\title{
IMAGE RETRIEVAL USING LANDMARK INDEXING FOR INDOOR NAVIGATION
}

\author{
by \\ DWAIPAYAN SINHA \\ A thesis submitted to the \\ Department of Electrical \& Computer Engineering \\ in conformity with the requirements for \\ the degree of Master of Applied Science
}

Queen's University

Kingston, Ontario, Canada

April 2014

Copyright @ Dwaipayan Sinha, 2014 


\begin{abstract}
A novel approach is proposed for real-time retrieval of images from a large database of overlapping images of an indoor environment. The procedure extracts visual features from images using selected computer vision techniques, and processes the extracted features to create a reduced list of features annotated with the frame numbers they appear in. This method is named landmark indexing. Unlike some state-of-the-art approaches, the proposed method does not need to consider large image adjacency graphs because the overlap of the images in the map sufficiently increases information gain, and mapping of similar features to the same landmark reduces the search space to improve search efficiency. Empirical evidence from experiments on real datasets shows high (90-100\%) accuracy in image retrieval, and improvement in search time from the order of 100-200 milliseconds to the order of 10-30 milliseconds. The image retrieval technique is also demonstrated by integrating it into a $3 \mathrm{D}$ real-time navigation system. This system is tested in several indoor environments and all experiments show accurate localization results in large indoor areas with errors in the order of 15-20 centimeters only.
\end{abstract}




\section{Statement of Co-Authorship}

The work presented in this thesis was accomplished under the supervision of Dr. Michael Greenspan, who provided feedback and corrections to the manuscript. The contents were accepted for publication in a conference paper cited here, which was coauthored by my colleague and Ph.D. student Mirza Tahir Ahmed and Dr. Greenspan.

- Dwaipayan Sinha, Mirza Tahir Ahmed, and Michael Greenspan. Image retrieval using landmark indexing for indoor navigation. Accepted at 2014 International Conference on Computer and Robot Vision. 


\section{Acknowledgments}

First and foremost, I would like to thank my supervisor Prof. Dr. Michael Greenspan for providing the opportunity to work under him, and for his wisdom and guidance while supervising my thesis research.

Secondly, I am thankful to all the members of the Robotics and Computer Vision Laboratory (RCVLab). In particular, I would like to acknowledge Mirza Tahir Ahmed, Salar Awan, Mustafa Mohammad, Kevin Hughes, Andrew Yaworski, Edith Deretey and Bipin Upadhyaya (Software Re-engineering Lab) for their advice and assistance in producing this thesis. I would also like to thank Paul Mrstik and Kresimir Kusevic from GeoDigital International Inc., Ottawa for their guidance in successfully finishing the project.

Finally, I am grateful to my friends and family for all their support and encouragement, with special thanks to Archita Mazumder. Lastly, I would like to thank my parents, Dipankar Sinha and Jayati Sinha for all their endless love, advice, and support throughout my academic career. 


\section{Contents}

Abstract $\quad$ i

Statement of Co-Authorship ii

$\begin{array}{ll}\text { Acknowledgments } & \text { iii }\end{array}$

Contents $\quad$ iv

List of Figures $\quad$ vi

List of Tables viii

Glossary $\quad$ ix

Chapter 1: Introduction 1

1.1 Background ........................ 1

1.1.1 Indoor Navigation . . . . . . . . . . . . . . . . . 1

1.1.2 Navigation with Landmarks . . . . . . . . . . . . . . 2

1.1.3 Application of Computer Vision: Image Retrieval and Visual Words . . . . . . . . . . . . . . . . 3

1.1.4 Integration to 3D Processing . . . . . . . . . . . . . 5

1.2 Problem Identification . . . . . . . . . . . . . . . . 5

1.3 Thesis Contribution . . . . . . . . . . . . . . 6

1.4 Thesis Outline . . . . . . . . . . . . . . . . . . 7

Chapter 2: $\quad$ Literature Review 9

2.1 Image Retrieval using Features . . . . . . . . . . . . . . . . . . 9

2.1.1 Feature Extraction . . . . . . . . . . . . . . 10

2.1.2 Feature Matching . . . . . . . . . . . . . . . . . 15

2.2 Bag of Visual Words and Related Work . . . . . . . . . . . . . . . 17

2.2.1 Feature Quantization . . . . . . . . . . . . . 18

2.2.2 Bag of Visual Words Approach . . . . . . . . . . . . . . . 18 
2.2 .3 Proposed Extensions _. . . . . . . . . . . . . . . . . . 21

2.3 Localization . . . . . . . . . . . . . . . . . . . . . . . . 26

2.3.1 Direct 3D Registration . . . . . . . . . . . . . . . 26

2.3 .2 Image-based Localization . . . . . . . . . . . . . . . . . . . . 28

2.4 Discussion . . . . . . . . . . . . . . . . . . . . . . . . . . . . 33

$\begin{array}{ll}\text { Chapter 3: } & \text { Landmark Indexing } \\ 36\end{array}$

3.1 Problem Formulation . . . . . . . . . . . . . . . . . . . 36

3.2 Landmarks . . . . . . . . . . . . . . . . . . . . . . . . . . . . 38

3.3 Landmark Index Generation . . . . . . . . . . . . . . . . . . . . 39

3.3 .1 Image Acquisition . . . . . . . . . . . . . . . . . . . . 39

3.3 .2 Implementation . . . . . . . . . . . . . . . . . . . . 40

3.4 Search Technique . . . . . . . . . . . . . . . . . . . . 43

3.5 Demonstration in 3D Navigation . . . . . . . . . . . 46

3.5 .1 Pre-processing . . . . . . . . . . . . . . . . . 46

3.5 .2 Runtime . . . . . . . . . . . . . . . . . . . 49

Chapter 4: Experimental Results $\quad 53$

4.1 Data Acquisition . . . . . . . . . . . . . . . . 53

4.2 Filter Parameter Estimation . . . . . . . . . . . . . . . 57

4.3 Landmark Index Generation Results . . . . . . . . . . . . . . . . 59

4.4 Search Accuracy and Performance . . . . . . . . . . . . . . 61

4.5 Indoor Navigation . . . . . . . . . . . . . . . . . . . . . 69

$\begin{array}{lll}\text { Chapter 5: } & \text { Conclusions and Future Work }\end{array}$

5.1 Summary of Conclusions . . . . . . . . . . . . . . . . . 72

5.2 Future Work . . . . . . . . . . . . . . . . . . . . 73

$\begin{array}{ll}\text { Bibliography } & 75\end{array}$ 


\section{List of Figures}

2.1 Block Diagram of SIFT . . . . . . . . . . . . . . . . . 12

2.2 Scale-space Extrema Detection . . . . . . . . . . . . . . . . . 13

2.3 SIFT Results . . . . . . . . . . . . . . . . . . . 15

2.4 SIFT Matching Example . . . . . . . . . . . . . . . . . . 17

2.5 Block Diagram of Bag of Visual Words . . . . . . . . . . . 20

2.6 Block Diagram of Image-based Localization _ . . . . . . . . . . . 29

3.1 Block Diagram of Landmark Index Generation Procedure . . . . . . . 41

3.2 Block Diagram of Image Search Procedure . . . . . . . . . . . . . 44

3.3 Block Diagram of Pre-processing . . . . . . . . . . . . . 47

3.4 Block Diagram of Runtime . . . . . . . . . . . . . . . . 50

4.1 Example Partial Dataset . . . . . . . . . . . . . . . . . 55

$4.2 \quad$ ROC Space Plot . . . . . . . . . . . . . . . . . . . . . . 58

4.3 True Positive Image Retrieval Examples _ . . . . . . . . . . . . . 64

4.3 True Positive Image Retrieval Examples (contd.) . . . . . . . . . . 65

4.3 True Positive Image Retrieval Examples (contd.) . . . . . . . . . 66

4.4 False Positive Image Retrieval Examples _ . . . . . . . . . . 67

4.5 Accuracy and Performance Comparison . . . . . . . . . . . 68

4.6 The Indoor Positioning System Setup . . . . . . . . . . . . . . . . 69 
4.7 Indoor Navigation in ECE Dept. Office . . . . . . . . . . . . . . 70 


\section{List of Tables}

2.1 SIFT Feature Matching with Different Methods . . . . . . . . . . . 17

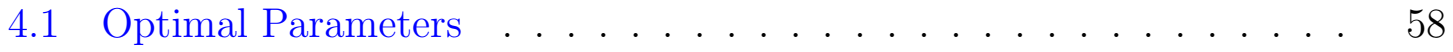

4.2 Landmark Index Generation Results . . . . . . . . . . . . . . . 60

4.3 Search Accuracy and Performance . . . . . . . . . . . . . . . . 62

4.4 RANSAC Registration Error in Navigation . . . . . . . . . . . . 71 


\section{Glossary}

2D Two dimensional.

3D Three dimensional.

DoF Degrees of Freedom.

DoG Difference of Gaussians.

FLANN Fast Library for Approximate Nearest Neighbor [49].

OpenCV Open source Computer Vision library for $\mathrm{C}++$ [8].

PCA Principal Component Analysis.

PCL Point Cloud Library [60].

RANSAC RANdom SAmple Consensus.

RGBDSLAM Open source software package for SLAM using RGB-Depth sensors [17].

ROC Receiver Operating Characteristic.

ROS Robot Operating System [53]. 
SIFT Scale Invariant Feature Transform.

SLAM Simultaneous Localization And Mapping.

SURF Speeded Up Robust Features.

TF-IDF Term Frequency - Inverse Document Frequency. 


\section{Chapter 1}

\section{Introduction}

\section{$1.1 \quad$ Background}

\subsubsection{Indoor Navigation}

Indoor navigation is the method of localizing a robot or any other user in an indoor or underground environment using a sensor or a network of sensors. Indoor navigation systems are designed not only for positioning, but for public aid and marketing purposes. They can be used to guide visually impaired people, to track packages in a large warehouse, arrange tours in educational or industrial campuses, or even navigate a robot inside abandoned underground mines. Location-based marketing technique such as targeted advertising can be integrated into such a navigation system.

Before the emergence of computer vision aided navigation, sensors like SOund Navigation And Ranging(SONAR), Radio-Frequency IDentification (RFID) etc. were used. SONAR sensors can be used to find the distance from walls using the time of flight of ultrasonic signal. RFID tags are cheap, can be read even if they are invisible, and multiple tags can be read at the same time. In long tunnelways, RFID markers can be placed at different places, so that when a vehicle comes near a marker, a 
controller can identify the location. The same can be applied in different floors or rooms of a building. Wi-fi is also another possibility. The intensity of the received signal, and identifying the wi-fi fingerprint are key aspects for this type of navigation. However, RFID tags can only be used when a tag comes close to a sensor, and wi-fi technology can indicate an area where a device is located instead of its pose in 6 Degrees of Freedom. Both technologies need a sufficient number of tags or access points throughout the map for accurate localization. To estimate the pose in a 3D model, some other information is required. The first applications of $3 \mathrm{D}$ vision in localization and mapping were done with laser scanners. They can be used to estimate roll, pitch and yaw, and full 6 DoF in pose estimation can be achieved.

\subsubsection{Navigation with Landmarks}

The challenge in navigation is different than Simultaneous Localization and Mapping (SLAM), mostly because mapping is not involved. In navigation, the idea is to locate a robot or a person in a map through searching the environment for at least one landmark. The approach of using landmarks in localization is derived from the human notion of navigation. A well-recognizable feature can be considered as a landmark of a certain region, like a geographic natural feature (e.g., Barringer Meteor Crater in Arizona, US), a well-known building in a city (e.g., Taj Mahal in Agra, India), or an indoor reference point like a reception desk in a hotel. In computer vision, features, or groups of features, are considered as landmarks. Se et al. [64] first assigned the term to visual features, and the idea is extended in this paper.

In SLAM, depending on the sensor, the landmarks can be artificial, i.e., added

externally to the environment. For example, previously discussed RFID markers 
are artificial landmarks added to different locations in a building or a long tunnel way. For vision-based navigation, artificial landmarks may or may not be necessary. One example of modern day artificial landmarks in vision-based navigation is Quick Response (QR) codes. All smartphones currently have the capability to read QR codes, and QR codes are specifically useful because any information can be converted into a code. So exact coordinates can be encoded and placed on a wall, and the smartphone can scan it and display the location. However, computer vision enables the positioning system to identify and make use of the landmarks which naturally exist in the environment.

\subsubsection{Application of Computer Vision: Image Retrieval and Visual Words}

Computer vision techniques have become relevant with the topic of navigation with the development of different feature extraction methods. Images can be identified using features. A feature point is a point which should be distinctive, invariant to image scaling, rotation, illumination or camera viewpoint, and robust to noise. There

are several algorithms to extract features from images, like SIFT [45], SURF [5], BRISK [38], FREAK [2] etc. Some algorithms provide only point locations in an image, and some algorithms provide a feature descriptor for the each of the points. A feature descriptor is a vector describing the unique properties of the corresponding feature point. SIFT and SURF have their own descriptors. FREAK produces only points, which can be described using descriptors like BRIEF [10] or ORB [57]. Image matching is performed in feature space. If two images have feature points described with the same algorithm, and the descriptor vector has $N$ dimensions, then in that $N$-dimensional space two features from different images closest to each other are 
considered a match. In a situation like navigation, a map can have several, maybe thousands of images. To identify a location, a user needs to capture a frame with a camera, and perform a feature matching with the images in the map. The image with most matches with the captured frame can present the location of the user.

There is a challenge of image identification for navigation. As stated before, a map dataset can have several thousands of images. Matching a frame with each one of them will certainly be a hindrance to real-time retrieval. A procedure is needed to reduce the search set. This motivates the work described in the thesis.

There exists a method called bag of visual words, first introduced by Sivic and Zisserman in 2003 [66]. An image can be compared with a document, which is an unordered collection or a bag of words. Image features, which are similar to each other according to some pre-defined criteria, can be classified as a visual word. When matching features to retrieve an image from a database, search is performed on these visual words, significantly reducing the search space. However, if image retrieval is performed for the purpose of navigation in a large map, all the features have to be taken into account to make the visual word dictionary or codebook, and some features may not be useful for identification. Moving and temporary objects like a car or people on the road cannot be taken as useful. Only distinguishable, repeatable and static features are considered favorable, and the rest should be filtered out. This requires a suitable algorithm, which is the central point of this thesis, and discussed in detail in chapter 3 . The good features which are not removed from the map database are treated as visual landmarks. 


\subsubsection{Integration to 3D Processing}

Current development of Point Cloud technology helps to describe a 3D model as a collection of 3D points, termed as a point cloud. Each point is described by its 3D coordinates with respect to a viewpoint. The coordinates are measured in distance units. There may be additional properties added to each point, such as image information like color and opacity, or geometric information like surface normals and curvature. Point Cloud Library (PCL) [60] discriminates the point clouds as either organized or unorganized. An organized point cloud has its points has no missing information from its $2 \mathrm{D}$ image equivalent. If a point cloud is projected on a plane perpendicular to the principal axis of the camera, then each point in the cloud can be addressed with the 2D row-column coordinates their corresponding pixel in the resultant projected image. For example, a $640 \times 480$ organized point cloud has all 307200 points, none missing, and each point can be referred with 2D row and column pixel coordinates (e.g., 0-639, 0-479). This is advantageous for 3D navigation. When an image is retrieved, if the corresponding point cloud is available, all the feature matches found in the retrieval step can be translated into 3D space. These 3D matches can be readily used to register 3D frames and find the position and orientation in 6 DoF.

\subsection{Problem Identification}

The existing solutions for location image identification using bag of visual words process a large number of features which cannot be considered as landmarks. This makes the localization process slow, and often introduces inaccuracies at runtime. Secondly, the existing approaches to reduce the amount of features have not considered the difference between outdoor and indoor environments. Features can be filtered using 
some criteria on the number of appearances in the image database, and imposing some overlap between adjacent images can be helpful to gain prior information to accurately match similar features.

\subsection{Thesis Contribution}

Proposed Approach. This thesis proposes a novel method which uses a feature indexing approach, which speeds up the retrieval process compared to the histogram matching of bag of visual words. Features from each image are extracted and duplicate features are removed, keeping only the image identification numbers they appear in. This reduced set of features are again passed through a bandpass filter to remove sporadic or too frequent features. The remaining features are considered as landmarks. During image retrieval, features from the query image are extracted and matched with the landmark database. Each of the training image numbers associated with the matched landmarks gets a vote, and the image number with the maximum votes is chosen as the retrieved image. The search procedure gets reduced to creating a one-dimensional array of integers and finding the maximum element. This is a significant enhancement in performance over bag of visual words where each image is represented as a histogram of word frequencies, and retrieval is implemented as a histogram matching procedure in a very high-dimensional space, which, due to the "curse of dimensionality", is a time-consuming process in practical approaches. It is also shown in this thesis that the proposed landmark indexing method has a complexity $\mathrm{O}(P N)$ which is very less than the $\mathrm{O}(M N)$ complexity of bag of visual words, where $P$ is the number of features in the query image, $M$ is the 
number of entries in landmark index and $N$ is the number of training images.

Experimentation. A number of quantitative experiments support that the proposed solution can accurately retrieve images from a large database very efficiently. The speed of identification is in real-time, far faster than current methods, while the accuracy is comparable to and sometimes better than those methods. In addition, the landmark indexing method is integrated into a modified RGBDSLAM framework [17] used for indoor navigation. Recorded values suggest that the navigation results are very accurate in large indoor environments, and the observed 3D registration errors are similar to the existing evaluation of the system, confirming that the image retrieval method correctly performs in any indoor navigation scenario.

\subsection{Thesis Outline}

The thesis is organized as follows:

Chapter 2, Literature Review: This chapter discusses different existing publications on vision-based localization and image retrieval algorithms.

Chapter 3, Landmark Indexing: This chapter describes the method to reduce image retrieval search space into a landmark index. The term landmark is defined, the procedure to identify landmarks is depicted, and how this algorithm can be further combined to 3D indoor navigation is explained.

Chapter 4, Experimental Results: Quantitative evidence in favor of the accuracy and speed of the proposed method is produced in this chapter. Comparison with existing approaches is shown using datasets from different types of indoor environments. Also, results of the application of landmark indexing in a 3D real-time 
navigation system is described in this chapter.

Chapter 5, Conclusions and Future Work: This chapter concludes the thesis, with a short introduction to the possible future extensions and applications of the landmark indexing approach. 


\section{Chapter 2}

\section{Literature Review}

\subsection{Image Retrieval using Features}

Image retrieval is the task of accurately matching a query image to another similar image of the same object or scene from a large set of images [72]. Image retrieval techniques may be generalized, or may have a special application, e.g., trademark, face, or text-based document retrieval. All these techniques have three main aspects in common [58]:

1. Feature Extraction: Extract visual features such as SIFT [45] or SURF [5], and store them in a database with the image dataset.

2. Indexing: Identify useful images or features based on some criteria and rank them accordingly. Some example approaches are keyframe detection $[16,30]$, and reducing the feature set based on similarity between images [72].

3. Retrieval: Capture the image, and search the database to find the image, or the set of images, with the greatest number of feature matches with the query image. 
Further explanation of existing feature extraction and matching methods follows.

\subsubsection{Feature Extraction}

Identifying features in an image is a crucial step in image matching. Each feature is an interest point in the image computed using intrinsic image properties, and a local descriptor which describes the interest point and its surrounding points. In most cases, this descriptor is a high-dimensional vector. Tuytelaars and Mikolajczyk [73] identified properties that an ideal feature must have. These are:

- Repeatability: Two images of same object must have a high number of common features. The features must be invariant to geometric and photometric transformations, and robust to disturbances, e.g., noise, blur, compression artifacts, etc.

- Distinctiveness: Features must be distinguishable so that they can be recognized in a large database of objects.

- Locality: Local features must be robust to occlusion and clutter.

- Quantity: The feature detector must always generate a sufficiently large number of dense features from even small objects

- Accuracy: The localization of the feature must be precise.

- Efficiency: The feature detection method must perform in real time.

There are many popular feature extractors, starting from Moravec's interest operator [48] and the Harris corner detector [28] to modern feature detection algorithms such as SIFT [44,45], PCA-SIFT [33], SURF [5], BRISK [38], BRIEF [10], ORB [57], 
and FREAK [2]. The following discussion is mainly about SIFT and its origins, because it is still a very widely popular feature extractor, and used by the method proposed in this thesis, and also other methods it is compared against.

One of the earliest interest point detectors was Moravec's interest operator [48]. He extracted interest points by assuming a patch on each pixel of the image, and then finding the similarity between each patch and its neighbor patches in every direction (horizontal, vertical, and two diagonals). The difference between patches are measured by the sum of squared differences of the patch pixels. If the minimum of the calculated differences is high enough, then the considered pixel is chosen as an interest point. Moravec's operator is obviously anisotropic (only directions at every $45^{\circ}$ is observed) and noisy (the patch around a point is binary and rectangular). An alternative was suggested by Harris and Stephens [28]. To overcome the anisotropic nature of Moravec, they considered the image gradient. The Harris matrix $H$ at a pixel $p$ is defined as:

$$
H=\left\{\begin{array}{cc}
\sum G_{x}^{2} & \sum G_{x} G_{y} \\
\sum G_{y} G_{x} & \sum G_{y}^{2}
\end{array}\right\}
$$

where $G_{x}$ and $G_{y}$ are the respective $x$ and $y$ Gradient magnitude images, and the summation is taken over all points in a neighborhood of $p$. The symmetric matrix $H$ can be subjected to principal component analysis to estimate eigenvalues $\lambda_{1}$ and $\lambda_{2}$. If both of the eigenvalues cross some threshold, then it shows strong variation in both direction, and the pixel $p$ is considered as an interest point.

The Harris corner detector was one of the most popular interest point detectors for the purpose of image matching. However, it was not scale-invariant. Automatic scale selection during interest point estimation was suggested by Lindeberg [41], and 


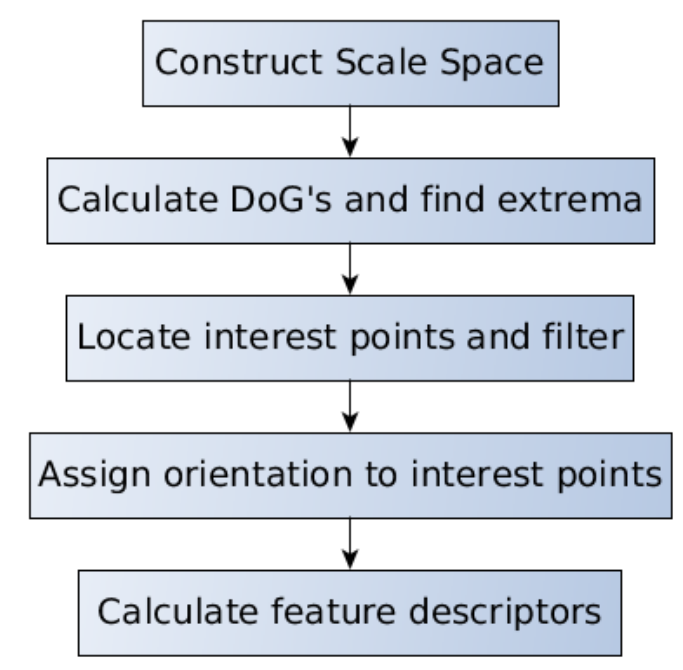

Figure 2.1: Block diagram of SIFT

this was further refined by Mikolajczyk and Schmid [47] with introducing the HarrisLaplacian operator. Their approach applies Harris corner detector at multiple scales, and then chooses the characteristic scale with the Laplacian.

Scale-Invariant Feature Transform (SIFT) was first coined in 1999 by David Lowe. Approximating the Laplacian of Gaussian (LoG) of the Harris-Laplacian operator with a Difference of Gaussian (DoG), he accelerated the performance. The complete procedure is shown in Figure 2.1.

The scale-space construction is performed using the Gaussian kernel provided by Lindeberg [40]. The DoG is computed by

$$
D(x, y, \sigma)=(G(x, y, k \sigma)-G(x, y, \sigma)) * I(x, y)
$$




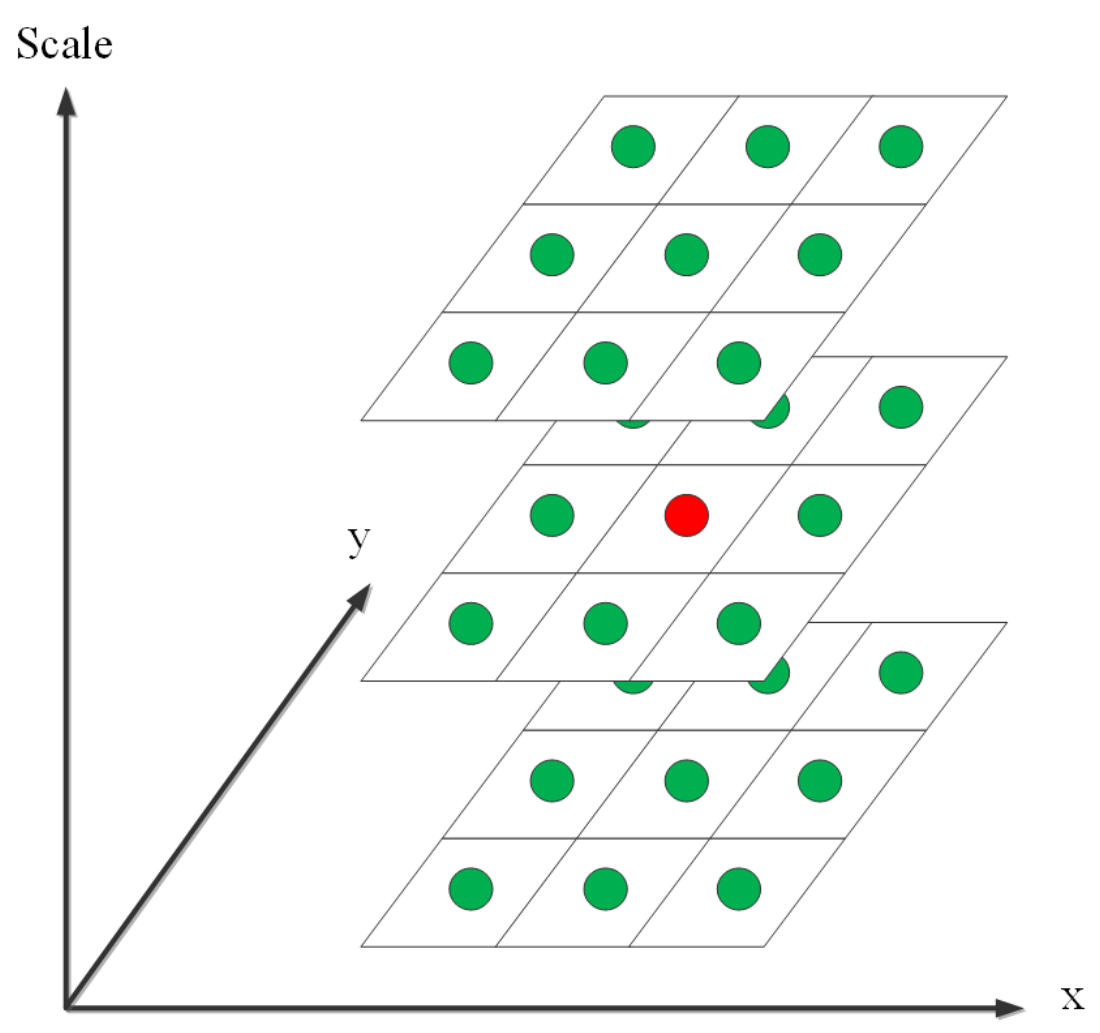

Figure 2.2: Detection of extrema in scale-space by comparing a pixel (the red circle at the central scale space) to its neighbors (the green circles) in the current and adjacent scales (based on [45]).

where $I(x, y)$ is the input image, * is the convolution operator, and $G(x, y, \sigma)$ is the Gaussian kernel, given by

$$
G(x, y, \sigma)=\frac{1}{2 \pi \sigma^{2}} e^{-\frac{x^{2}+y^{2}}{2 \sigma^{2}}}
$$

The local extrema of $D(x, y, \sigma)$ are detected by comparing each pixel to its eight neighbors in the current scale space level and the nine neighbors in the scale above and below. This is shown in Figure 2.2. The pixel for each detected extrema is chosen as a candidate to be an interest point. 
The filtering of the candidates is performed by expanding the $D(x, y, \sigma)$ function with Taylor Series approximation to quadratic terms, taking the derivative and setting it to 0 , thereby finding the extremum $\hat{\mathbf{x}}$. Then, all extrema with a value of $D(\hat{\mathbf{x}})$ less than a certain threshold are discarded, and principal component analysis helps to remove unstable localized points on edges, as the DoG will have a very large principal curvature compared to the small one in the perpendicular direction.

After the interest points are extracted, orientations are assigned to each of them based on the dominant gradient orientation of the surrounding patch. Then a representation of this image patch is estimated, which is the feature descriptor of the interest point. This representation is a local orientation histogram, containing the accumulated gradient magnitude of each sample point in the patch around the interest point. Generally, $4 \times 4$ arrays of histograms with 8 orientation bins in each histogram are computed from $16 \times 16$ sample arrays. This results in 128-dimensional vectors.

Speeded-Up Robust Features (SURF) [5] provides further approximation of Laplacian of Gaussian by using Box Filter. It also uses wavelet responses for orientation assignment and feature description. The advantage of using both box filter and wavelet responses is that these operations can be easily performed with integral images. Despite the fact this faster approach than SIFT was introduced in 2006, and even though there exist several other extensions and alternate methods [2,5,10,14,15,33,38,57,74], SIFT still seems to be the most popular, because it shows all of the ideal feature characteristics [73]. Figure 2.3 shows SIFT interest points in an image. For image retrieval from a location image database, which is the main goal of the proposed method in this thesis, SIFT features are seen to perform significantly better than other feature descriptors [77]. 


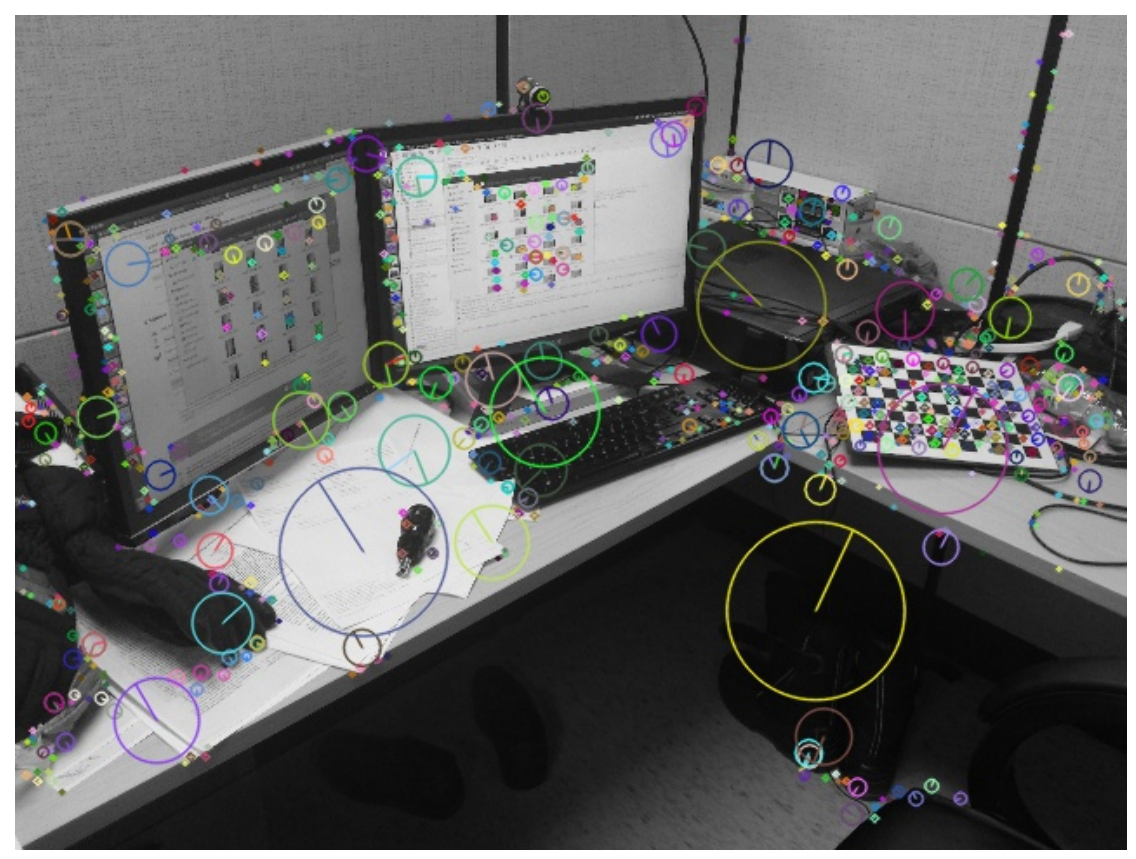

Figure 2.3: Detection of SIFT features in a scene

\subsubsection{Feature Matching}

Feature matching is performed by finding nearest neighbors in the high-dimensional descriptor space. There are several techniques to match features. The methods mostly used in this thesis are discussed here.

Linear Search. This is the most trivial method of matching features. Distance between a query feature and each of the training features is calculated, and the training feature with minimum distance is chosen as the nearest neighbor. If there are $m$ features in the query image, and $n$ features in training set, the time complexity of this method is $\mathrm{O}(\mathrm{mn})$. This is a costly mechanism, and arranging the training features in a data structure greatly improves performance.

$k$-D Tree. If the training features are organized in a $k$-d tree [21], then a depth-first search mechanism can find exact nearest neighbors in $\mathrm{O}(m \log n)$ time for a fixed 
dimension. However, performance is affected as dimensionality increases. Arya et al. [4] and Beis and Lowe [6] modified the algorithm by using early-stopping criteria, and Silpa-Anan and Hartley [65] proposed to use multiple randomized $k$-d trees to find approximate nearest neighbors to speed up the performance for high-dimensional features.

$k$-Means Tree. Arranging the training data in a tree generated by $k$-means clustering was first proposed in 1975 by Fukunaga and Narendra [22]. The tree construction is done by clustering data points into $k$ disjoint groups, and recursively performing the same task for each group. Nistér and Stewénius [51] used a similar tree for visual vocabulary construction, which is discussed later in Section 2.2.3.

FLANN. Muja and Lowe [49] proposed a method to automatically select an approximate nearest neighbor algorithm. Their algorithm can reduce either the tree building time, or reduce the time for searching. They compute a cost of each algorithm based on building time and memory consumption when applied on a given training dataset, and the algorithm with the lower cost is chosen. They released Fast Library for Approximate Nearest Neighbor (FLANN) with two algorithms, (1) randomized $k$-d tree [65] and (2) hierarchical $k$-means tree [22,51].

An example result of SIFT feature matching between two scenes is shown in Figure 2.4. The image in the left side has 1123 features, and the image in the right side has 852 features. Table 2.1 shows the time performance and matches found by each matching algorithm. 


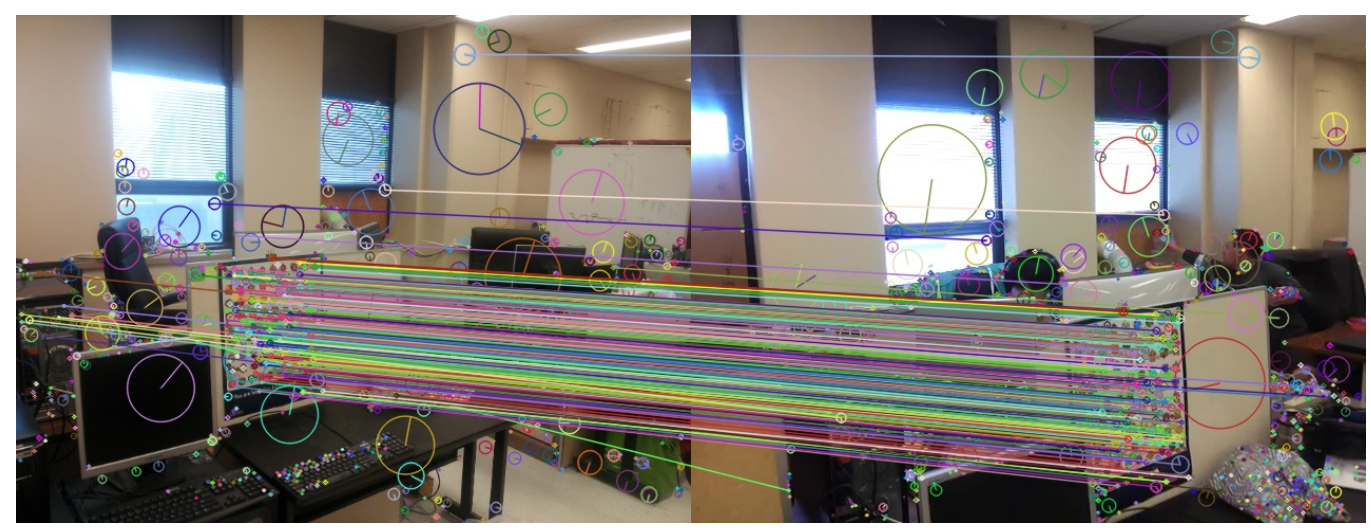

Figure 2.4: SIFT feature matching example

Table 2.1: Time performance and matches found by different methods matching SIFT features from the two images of Figure 2.4

\begin{tabular}{|c|c|c|}
\hline Method & Search time & Matches found \\
\hline$k$-D tree & $20 \mathrm{~ms}$ & 334 \\
\hline$k$-Means & $20 \mathrm{~ms}$ & 362 \\
\hline FLANN (Auto-tune) & $10 \mathrm{~ms}$ & 388 \\
\hline
\end{tabular}

\subsection{Bag of Visual Words and Related Work}

Storing an exhaustive set of features from all images of a map decreases the performance of the retrieval system as the number of training features increases. In 2003, Sivic and Zisserman proposed to represent an image as a bag of visual words [66]. This is comparable to the representation of a text document as a bag of words, where a bag means an unordered collection. According to Sivic and Zisserman, a visual word is a quantization of several feature descriptors. 


\subsubsection{Feature Quantization}

Each feature is a point, or a vector, in a high-dimensional space, and quantization through any clustering algorithm is possible. The most popular method for clustering features is $k$-means clustering [46]. This algorithm partitions $n$ features into $k<n$

groups. This $k$ is pre-determined by the user. The algorithm has multiple passes. On the first pass it randomly generates $k$ vectors as cluster means, and associates every other feature vector with the nearest mean. In each of the following passes, the centroid of each cluster becomes the new mean, and the feature association is again performed. The whole process is repeated until convergence is achieved. Mathematically, the objective is to minimize the error function

$$
E=\sum_{i=1}^{k} \sum_{f_{i j} \in C_{i}}\left(f_{i j}-\mu_{i}\right)^{2}
$$

where there are $k$ clusters $\left\{C_{1}, C_{2}, \ldots, C_{k}\right\}$ with cluster means $\left\{\mu_{1}, \mu_{2}, \ldots, \mu_{k}\right\}$, and $\left\{f_{i 1}, f_{i 2}, \ldots\right\}$ are feature vectors in the $i^{t h}$ cluster $C_{i}$.

This method is used to cluster SIFT descriptors into visual vocabularies in Bag of Visual Words.

\subsubsection{Bag of Visual Words Approach}

The features used by Sivic and Zisserman [66] are SIFT, each descriptor being a 128-D vector. The similarity of two descriptors can be evaluated by calculating their distance in 128-dimensional feature space. The distance measure used here is Mahalanobis distance. Between two features $f_{1}$ and $f_{2}$ of the same distribution with covariance 
matrix $S$, the Mahalanobis distance function is given by:

$$
\operatorname{dist}_{M}\left(f_{1}, f_{2}\right)=\sqrt{\left(f_{1}-f_{2}\right)^{T} S^{-1}\left(f_{1}-f_{2}\right)}
$$

Using this distance measure, the features are clustered using a $k$-means clustering algorithm. The choice of number of clusters $k$ is done empirically to optimize performance. Automatic algorithm configuration from Fast Library for Approximate Nearest Neighbor [49] is used here. The resultant cluster regions are the visual words, and are represented by the cluster means $[66,76]$. During query, a term-frequency inverse-document-frequency $(t f-i d f)$ ranking scheme, which is seen in text retrieval and document identification, is used to rank the images. If there are $n$ words in the vocabulary, and a total of $N$ images, then the $t f-i d f$ weight for $i^{\text {th }}$ word in the $j^{\text {th }}$ image is given by:

$$
t_{i j}=\frac{x_{i j}}{\sum_{i} x_{i j}} \log \frac{N}{\sum_{j} x_{i j}}, i \in[1, n], j \in[1, N]
$$

where $x_{i j}$ is number of times $i^{t h}$ word appears in the $j^{\text {th }}$ image. Every image including the query is represented by a vector of $t f-i d f$ weights $\left\{t_{1}, t_{2}, \ldots, t_{n}\right\}$. The scalar product of each vector from training images with that of the query image is calculated, and image retrieval is performed by finding the image corresponding to the maximum of these scalar products. Sivic and Zisserman retrieved shots from a movie using this approach. Yang et al. modified the approach by replacing the features descriptors from vanilla SIFT to PCA-SIFT [33], which gives 36-dimensional vectors instead of 128.

The general framework to create a bag of visual words vocabulary is shown in the 


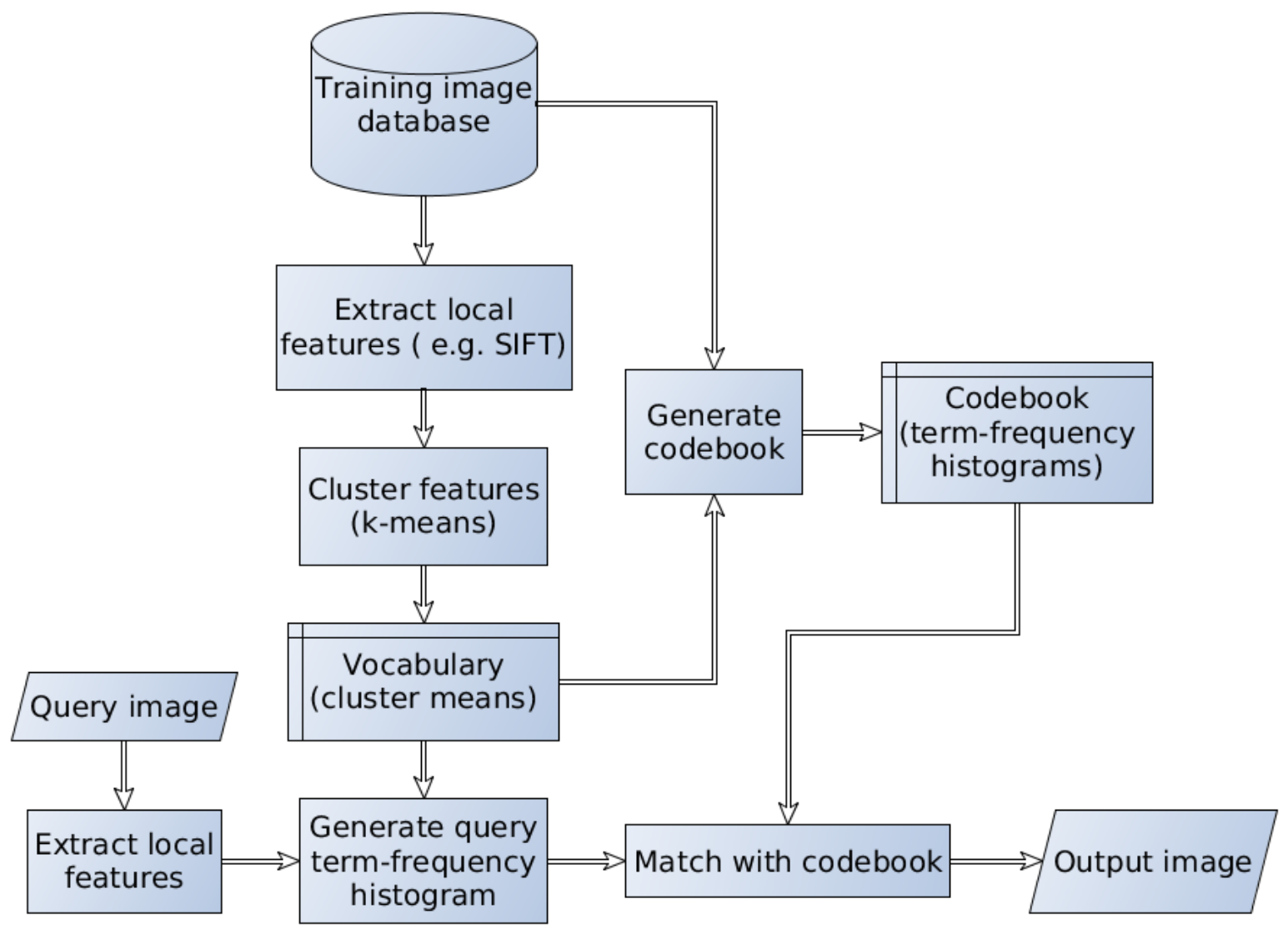

Figure 2.5: Block diagram of Bag of Visual Words

figure 2.5 .

If $k$-d tree is used to search each word in the features from query image, then for a vocabulary size $M$ and a training image database size $N$, to perform a single query, it takes:

- $\mathrm{O}(M \log P)$ time to build the term frequency histogram for the query image $(\mathrm{O}(\log P)$ for each word $)$, where $P$ is the number of features in the query image;

- $\mathrm{O}(M N)$ time to find the scalar product of the query histogram with those from the $N$ training images (linearly traversing the database), and find the largest 
scalar product.

\subsubsection{Proposed Extensions}

Tirilly et al. [70] and Yang et al. [76] compared different variations of the bag of visual words methods. Tirilly et al. described different forms of $t f-i d f$ weighting schemes. Yang et al. further proposed different feature selection criteria. They also showed the effect of different weighting schemes in accuracy and performance. The weighting schemes can be summarized into:

- Binary weight: 1 if a term is present in an image, 0 if not;

- Term frequency: Same as $x_{i j}$ from Equation 2.6;

- Normalized term frequency: $\frac{x_{i j}}{\sum_{i} x_{i j}}$;

- Term frequency - inverse document frequency: $t_{i j}$, which is defined in Equation 2.6;

- Normalized tf-idf: $\frac{t_{i j}}{\sum_{i} t_{i j}}$.

Yang et al. [76] noted that normalization is not preferable, because it reduces the distinctiveness of images as the effect of eliminating difference between similar visual words appearing in images of two areas differing in feature density. They also showed that binary weighting outperforms $t f-i d f$ for large training databases.

Subsequent publications using the idea of bag of visual words talk about creating a visual vocabulary and recognizing patterns with it. In 2006, Nistér and Stewénius [51] proposed a hierarchical $t f-i d f$ scoring using hierarchically defined visual words that form a vocabulary tree. The use of a tree structure for feature matching comes 
from David Lowe [45], who used a best-bin-first $k$-d tree to find approximate nearest neighbor to SIFT features. The vocabulary tree of Nistér and Stewénius is basically a hierarchical $k$-means tree. The training features are clustered using $k$-means clustering, the cluster means being the leaf nodes. Then, $k$-means clustering is re-applied on this set of cluster means to generate bigger clusters that are fewer in number. When assigning a feature to a visual word, search begins at the root, moves through branches containing close matches and ends when the leaf node with the closest matching visual word is found.

The vocabulary tree method was later adopted by Schindler et al. [62] in 2007. Their database consists of images with a considerable amount of overlap, so that the same areas are re-observed from different viewpoints. This knowledge of the overlapping frames helps to identify when the same visual words occur in multiple images, thus aiding in recognizing the images of the same location. This concept can be compared to the concept of information gain. If two random variables, $L$ and $W$, following a Bernoulli distribution, are defined on the event of being present on a certain location $l$ and the visibility of a particular visual word $w$ respectively, then the information gain on $w$ being visible at location $l$ is given using the entropy function $H()$ :

$$
I G(L, W)=H(L)-H(L \mid W)
$$

where the entropy function $H(X)$ for a random variable $X$ is defined as

$$
H(X)=-\sum_{x} P(X=x) \log [P(X=x)]
$$


and the conditional entropy is given by

$$
\left.H(Y \mid X)=-\sum_{x} P(X=x) H(Y \mid X=x)\right]
$$

Schindler et al. showed that $H(L)$ is constant for all visual words at location $l$, so to maximize the information gain, minimizing of the conditional entropy $H(L \mid W)$ is required. This conditional entropy, and therefore the information gain, can be expressed as a function of the $N_{w, l}$ (number of times $w$ appears at location $l$ ) and $N_{w, \bar{l}}$ (number of times $w$ appears everywhere else). The maximum value of information gain in equation 2.7 can be reached when $N_{w, \bar{l}}$ is minimized.

Further, [62] presents a generalization of the search algorithms for vocabulary trees. This algorithm is named Greedy N-Best Paths (GNP) algorithms, which can proceed on multiple branches at each level rather than finding the branch containing features closest to the query one.

Philbin et al. [52] improved the performance of searching in visual vocabulary using approximate nearest neighbor search algorithms. They compared their method, named approximate $k$-means clustering, with hierarchical $k$-means tree as proposed in [51]. The approximate $k$-means tree does not find exact nearest neighbor matches of features and cluster centers, but rather employs a forest of multiple randomized $k$-d trees built on each cluster center on every iteration. This reduces the complexity of standard $k$-means algorithm from $\mathrm{O}(n k)$ to $\mathrm{O}(n \log k)$, where $n$ is the total number of features. Experimental results in [52] show that this method outperforms the hierarchical $k$-means tree. Also, they incorporated a spatial verification in the ranking system by estimating the transformation between query and target images. 
Fraundorfer, Stewénius and Nistér [20] discussed how to overcome the limits of a computer being used to search an image from a large dataset. They proposed a binning scheme for fast hard drive search. Each of the bins represents a similar set of images stored in the hard disk. The search mechanism is hierarchical, i.e., the visual words from a query image are first looked up in the bins, and then the identified bins are fetched from the hard disk to retrieve the actual image. To find matches between features, they use Locality Sensitive Hashing (LSH) on binary descriptors.

Jégou et al. [32] stated that the approximate nearest neighbor matching techniques for visual words have some disadvantages. For a large visual dictionary, many true matches can be missed, and for a small dictionary, many false matches can appear. Hamming embedding solves the problem by computing a binary signature for a descriptor and incorporating the hamming distance of binary signatures of two features in nearest neighbor finding. Also, integrating a weak geometric consistency verification helps to improve the image ranking method of previous approaches. It is a low cost approach and can be applied to all images, where previous geometric verification approaches short-list only a few hundred images from a large dataset.

The concept that not all images or features are useful in matching a query image to a large dataset, is explored by Turcot and Lowe [72]. They label some of the features in the image database as useful, which are robust and distinctive. They use each of the images in the database as a query, and rank each image according to the similarity between the query and itself. This ranking helps to construct an image adjacency graph $G=(V, E)$, in which every image is represented as a vertex $v_{l} \in V, l \in\{1, N\}$, and each edge $e_{a b} \in E, a \neq b$ represents adjacency between $v_{a}$ and $v_{b}$. Each vertex is described with feature descriptors present within itself and also 
those from its neighboring images. This affects the $t f-i d f$ weights. In equation 2.6, $x_{i j}$ 's can be replaced with:

$$
y_{i j}=x_{i j}+\sum_{\substack{k \\ e_{j k} \in E}} x_{i k}, i \in[1, n], j \in[1, N] .
$$

The number of similar descriptors common to two adjacent images adds weight to the edge between them. Retrieving an image follows the same method. When a query image comes, it ranks the database images according to feature similarity and finds the nearest vertex to the query.

Knopp et al. [34] presented a very similar work. Their method approaches to recognize a place in a query image by matching with a city-wide database of street side images. They acknowledge the fact that there can be a lot of similar objects like trees, road markings, cars etc. to introduce confusion. However, they use the advantage of geotags present in the images to rule out confusing objects.

In 2010, Gavves and Snoek [23,24] defined visual synonyms. They use a similar idea to [72] and rank the images according to query-training feature similarity, only to further process the top ranked retrieved images for a geometric verification. Geometric connectivity is determined by estimating a homography matrix. Two matched features, mapped to different visual words, but consistent in geometric location, makes the corresponding word pair a candidate for visual synonyms. Word pairs that occur very rarely are rejected, and the remaining candidates are recognized as visual synonyms. The top ranking images containing the visual synonyms, and therefore become the result images of the retrieval process. 


\subsection{Localization}

There are two different approaches to register 3D frames to find the transformation matrix between them. The first approach is direct 3D registration, which involves extraction of 3D features from point clouds, the other one is to find 2D features in images, find matches, and translate the 2D matches to 3D.

\subsubsection{Direct 3D Registration}

The steps to perform direct 3D registration of two point clouds are similar to 2D image matching. They are:

1. Feature Extraction: Extract 3D features $[59,79]$ from point clouds. These 3D features, in contrast to 2D features which rely on intensity, are computed using surface normals and curvature. This is the main bottleneck of this approach. The surface normals on each point in the point cloud must be known, which takes the longest time to compute. For an noisy sensor like Kinect [3], each cloud must undergo pre-processing for noise removal. To increase computation efficiency, the point clouds can be downsampled, but it sacrifices accuracy of registration.

2. Feature Matching: Match features using methods previously described in 2.1.2.

3. 3D Transformation Estimation: Use RANSAC to calculate the camera pose matrix with respect to the world coordinate system using the correspondences.In the RANSAC method, three correspondences are selected, and the 6 DoF rigid transformation is calculated. Then, the re-projection error is found, and if it is 


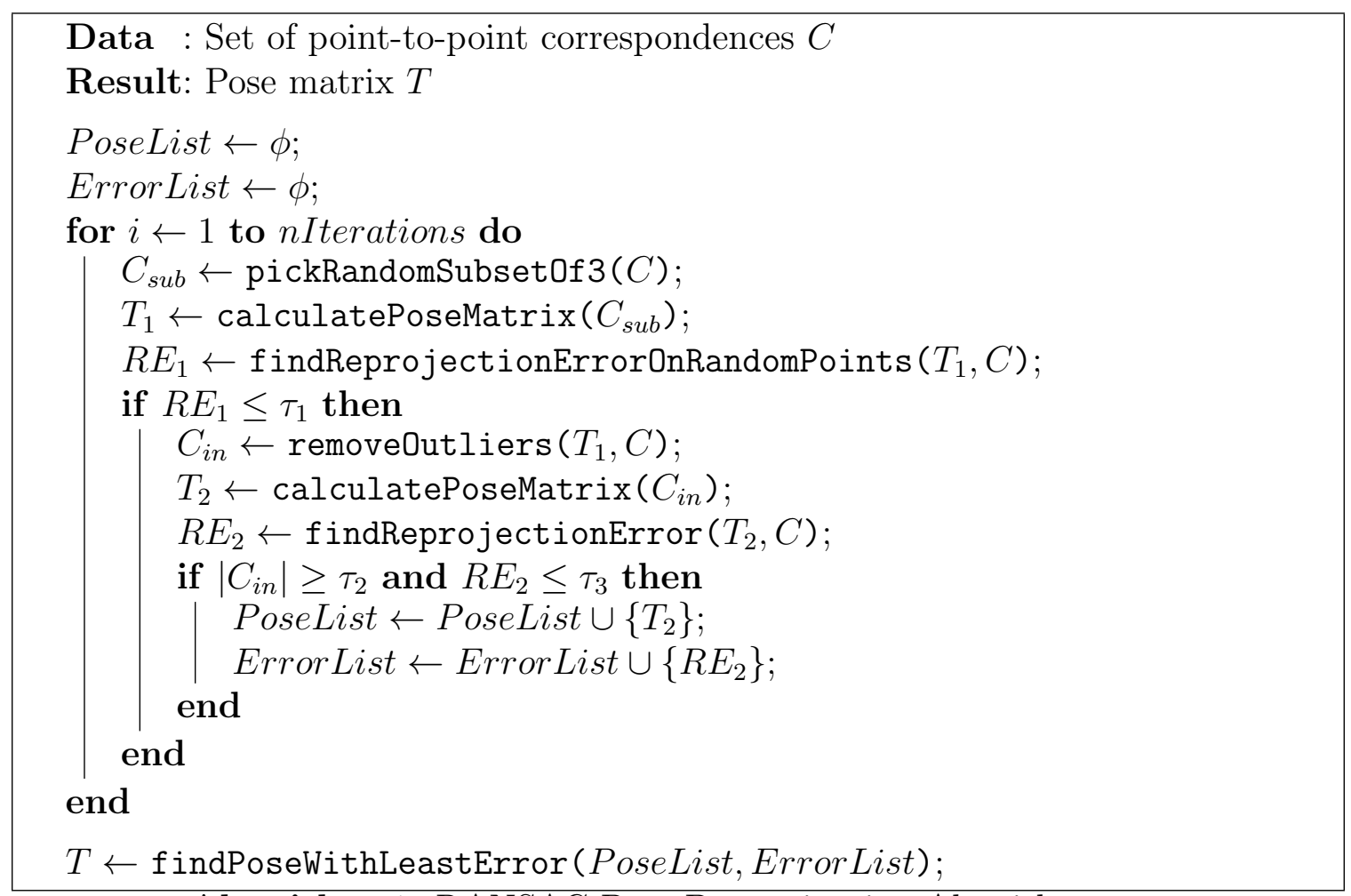

Algorithm 1: RANSAC Pose Determination Algorithm

too high, then the chosen correspondences are rejected. This approach removes any false matches. This process is repeated, until a suitable match is found, or an iteration threshold is exceeded. This best match is determined from the number of inliers being over a certain threshold and the re-projection error being below the tolerance level. This best pose is saved. The whole process is again repeated a pre-determined number of times and the best pose matrix is chosen from all those iterations. The algorithm is given as pseudocode in Algorithm 1. 


\subsubsection{Image-based Localization}

Image-based localization is the process of determining the exact location where a query image is taken [61]. If only the general image location is needed, then performing simple image retrieval is sufficient. If both the $3 \mathrm{D}$ position and orientation in 6 Degrees of Freedom are to be estimated, then either direct 3D frame acquisition with some sensor like LIDAR, or reconstruction of 3D model with Structure From Motion (SFM) approaches $[1,13,19,67,69]$ is required. If correspondences between 2D features and 3D points are given, the camera position and orientation can be computed [29]. Direct registration of 3D frames instead of 2D image matching is also used, but it is more memory and time consuming, while image retrieval is possible in real time.

In Simultaneous Localization and Mapping (SLAM), image retrieval techniques can be used to identify loop closure. Loop closure is closed traversal of an environment, i.e., the start location is re-visited during traversal, creating a loop. When loop closure is achieved, the localization error can be minimized and propagated throughout the entire map. Since image retrieval can be performed in real time, it is preferred over 3D matching techniques. Navigation in a pre-existing map is actually a repeated loop closure check.

The basic steps of image-based localization are:

1. Image Retrieval: Capture an image with a sensor, and retrieve the map image which is the closest match with the query image.

2. Matching: Use RANSAC to identify inliers in the correspondences of the query and retrieved images. 


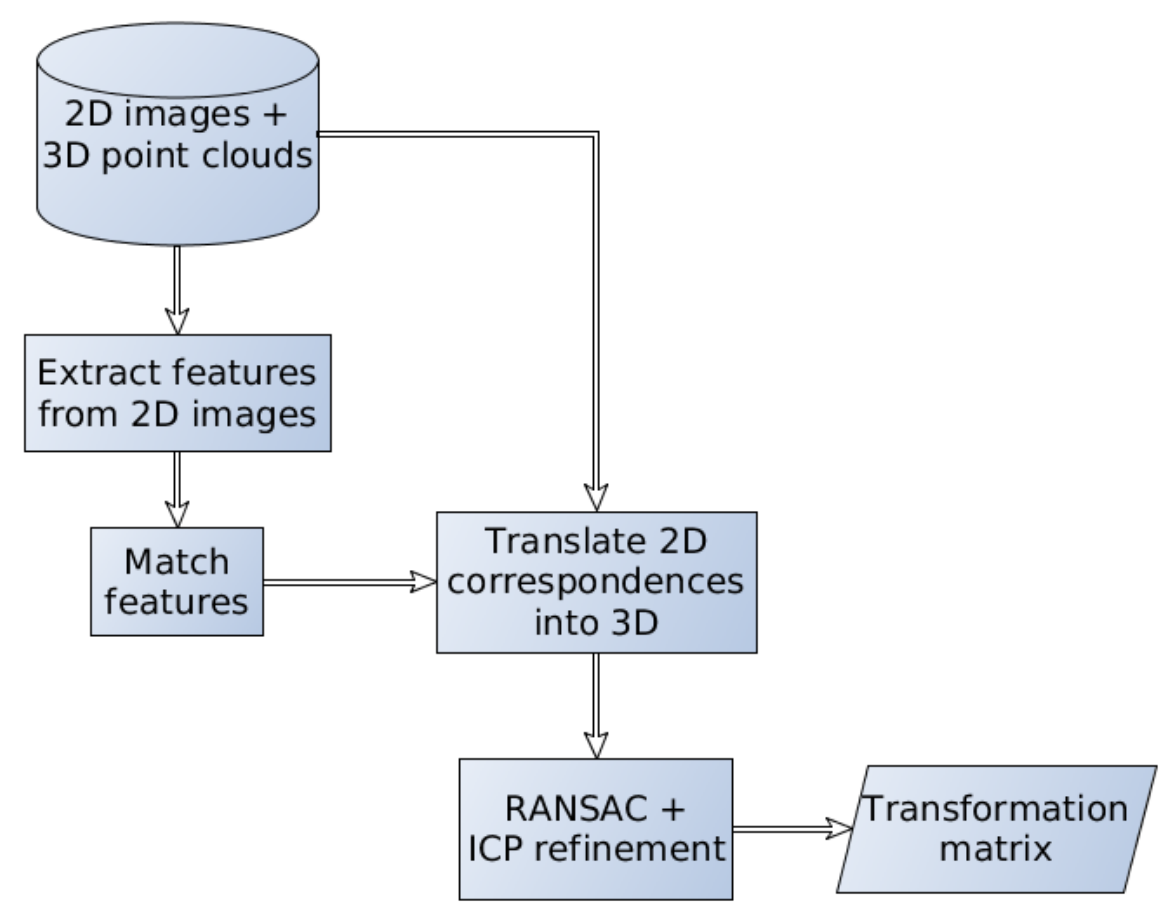

Figure 2.6: Block diagram of image-based localization, adapted from Henry et al. [31]

3. 3D Transformation Estimation: Acquire 3D frame from the sensor, translate the $2 \mathrm{D}$ correspondences from previous step to $3 \mathrm{D}$, and use them to calculate the camera pose matrix with respect to the world coordinate system. Iterative Closest Point algorithm [7] can be used to refine this RANSAC estimated pose matrix [31].

The complete procedure is presented as a block diagram in Figure 2.6.

\section{Related Works}

In vision-based navigation, feature-based location detection works best in a dense feature-rich environment, such as urban corridors, or indoor areas. Image retrieval techniques are developed to identify key locations in query images. Large image 
databases of places can be stored in the system as individual images, or as features extracted from those images to reduce the storage space [45]. Once the image is retrieved, it can be verified by fetching the corresponding 3D organized point clouds and finding the orientation and translation of the camera in 6 Degrees of Freedom. This method of verification is discussed in detail in chapter 3 and results are presented in Chapter 4.

One of the first works on vision-based localization and mapping techniques is done by Se, Lowe and Little [63]. The method describes tracking of SIFT features [45], and estimation of 3D location with the help of Triclops [50], a trinocular stereo camera system. They perform a stereo matching algorithm on the images from the left and right cameras, and use the top camera to impose constraints on the matching. The robot movement is tracked through tracking of the features, or landmarks. This approach of using features as landmarks is further expanded in the work described in this thesis (see section 3.2).

Earlier approaches $[54,78]$ in image retrieval-based navigation involved comparing query images with an exhaustive set of training images. This method was introduced by Robertson and Cipolla [54]. In their approach, a map is a database of photographs of multiple buildings in an area. The first step in localization is to perform image retrieval on the database using vertical vanishing direction for alignment of a building view in the query image to the canonical view in the database. It is followed by the pose estimation using point feature matching. Zhang and Kosecka [78] proposed a similar method, retrieving image by matching of SIFT features, and estimating the motion by computing the essential matrix between the query and best-matched reference images, and finally calculating the pose from triangulation. 
David Filliat [18] proposed an incremental bag of visual words approach for localization. He used a classifier on the words extracted from images. The classifier can be a Support Vector Machine (SVM) or a Bayesian classsifier. The incremental training method of the classifier helps to add new classes of words from new queries without processing old set of words. He performs experiments using different features such as SIFT and local color histograms. The distance between the feature vectors are replaced from Mahalanobis distance (equation 2.5) to chi-square distance:

$$
\operatorname{dist}_{\chi^{2}}\left(f_{1}, f_{2}\right)=\frac{\left(f_{1}-f_{2}\right)^{2}}{f_{1}+f_{2}}
$$

Henry et al. [31] proposed the idea of using both RGB and depth data for frameto-frame alignment of point clouds and loop closure detection. The proposed method extracts sparse features from RGB and depth images, and creates the dense point cloud in parallel. SIFT point features are extracted from each of the frames, and RANSAC is performed over these two sets of point features. This alignment is then refined using ICP. To minimize the error between the matched pairs of dense points, a Levenberg-Marquardt optimizer is used on a point-to-plane error metric along each target point's normal. The concept of keyframes is also introduced here. These keyframes are determined using the RANSAC inlier threshold, and every time a keyframe is created it is matched with all the previous keyframes for a loop closure detection. A graph optimization technique TORO [26,27] is employed to minimize the conflict between sequential constraints and loop closure constraints. This work is extended [30] in 2012. The Euclidean-space RANSAC is replaced with frame-toframe alignment RANSAC, SIFT feature points and descriptors are replaced with FAST features $[55,56]$ and Calonder descriptors [9], TORO is replaced with Sparse 
Bundle Adjustment [35,43,71], and the efficiency of loop closure detection is improved with place recognition. Here the method of Nistér and Stewénius [51] of creating a hierarchical visual word vocabulary tree is used.

Du et al. [16] also proposed combining RGB and depth data to form a 3D model. They partition the registration process into local alignment and global alignment. This is actually similar to [31] because local alignment handles the frame-to-frame registration, and global alignment uses loop closure information to optimize the poses of the frames to make them consistent with respect to a world reference frame. The idea of keyframes is applied here to detect the loop closure. The difference between [31] and this method is that Du et al. introduced a user feedback system for error handling. The system can inform the user if there is any error in registration, and the user can rewind and restart the process. When a loop closure is detected, the user can verify it through visualization. This visualization component also helps the user to check if there is any incomplete area in the map, and visit corresponding areas to capture frames and fill those holes. For the registration procedure, they use RANSAC to determine the rotation-translation vector pair, then apply a visibility conflict check with the inlier threshold. This visibility conflict is actually a verification that, if two cameras have an overlapping view of a plane, then after pose determination, the overlapped area must appear on the same image plane for both cameras. If points on that overlapping plane observed by camera 1 exist between the image plane of camera 2 and the camera 2 itself, then a conflict arises. In case of such conflict, the registration is considered incorrect and is reported to the user.

In a paper published in IPIN 2013, Liang et al. [39] described a method to use image retrieval to navigate in a shopping mall environment with a portable backpack 
arrangement of sensors $[12,42]$. They proposed the idea of storing features instead of images in the map database. They extract SIFT features from all the training images, and store them in a data structure with the indexes of images where each feature appears. When a query image is captured with the camera being used for navigation, all the features are extracted from that query image and matched using a $k$-d tree. A geometric consistency check is applied over the found matches, and after computing the fundamental matrix between the database and query images, the feature matches which do not follow the epipolar constraint are removed. The image from which most features are matched is identified. The roll and pitch are retrieved from cell phone sensors, and yaw is estimated. RANSAC is applied to find the translation between query and database images. The whole process may take $4-14$ seconds. Now a confidence estimation procedure is employed, which measures retrieval confidence and pose confidence. The retrieval confidence relies on the number of geometrically consistent feature matches between query and retrieved images. The pose confidence is based upon the number of inliers in RANSAC homography computation and reprojection error.

\subsection{Discussion}

The bag of visual words presents a solution for reducing the set of features of the map. It is very useful, but also an approximation of direct matching and decreases matching accuracy compared to the brute-force approach. Moreover, the term frequency histogram matching in a very high dimensional space (the dimension of the histogram is same as the number of training images) greatly affects performance. Some of the other described extensions of this method try to speed up the process by improving 
feature-visual word assignment, or improving the search mechanism [32]. Mapping multiple features to a single visual word also helps, but this may show no increase in accuracy, and with increasing number of features mapped to the same word, accuracy will certainly decrease. A more accurate matching and assignment process is therefore needed.

Schindler et al. [62] justified the use of overlapping images of an environment in recognizing visual words from the same location appearing in different images. This concept is used in the proposed method in this thesis, where the images acquired must be overlapping.

The Turcot-Lowe method [72], as described before, removes useless features with some ranking of the images and creating an image adjacency graph. However, there is a difference of assumption in the method proposed in this thesis. The agreement on the observation, that the number of useful features in a dataset is a small fraction of the total number of features, is the only similarity between both approaches. The difference lies in the targeted environment. As said earlier, the Turcot-Lowe method finds landmarks in buildings in a city area. Most large scale landmark image search methods [11, 23-25, 32, 34] work with this assumption. In an outdoor environment with wide-angle shots of the buildings, the number of stationary and re-observable features found will be small. People, cars or other non-landmark objects occupy most areas of the images taken in their dataset. This is not the case when performing navigation in an indoor context. The mapping is done systematically, with the images having a sequence rather than arbitrary images of different corners. So the burden of the image graph generation is not required. Also, in this scenario, the number of stationary objects is much greater. Features from desks, tables, windows, doors, 
posters on the wall, everything can contribute in the landmark database. From this observation, it can be assumed that culling of features applied to an indoor map will not be able to eliminate as much as $96 \%$ of the features which is shown in [72], and this is confirmed by experimental results presented in the following chapter 4 .

In the recently proposed method of Liang et al. [39]. They have presented the idea of image retrieval, but it has several drawbacks. First, although the data structure storing feature described is similar to the one proposed in this thesis, they use an exhaustive set of feature descriptors. They do not remove anything that can be a potential non-landmark. Secondly, the search mechanism employed was $k$-d trees, where there are other techniques like $k$-means clustering which can provide much more accurate and fast matches between features. The employment of a geometric consistency matching technique during runtime makes Liang's approach take 2-4 seconds in the retrieval steps alone, and far more for the pose estimation. This is unacceptable in a GPS style localization system, which may be used as a guidance system for visually handicapped people, or automated robots designed for fast response. The conducted experiments depicted in chapter 4 show that the landmark indexing method retrieves images in real-time, which aids in real-time navigation. 


\section{Chapter 3}

\section{Landmark Indexing}

\subsection{Problem Formulation}

The problem of image retrieval can be defined as:

Find the set of images $R$ given a query image $q$, such that:

$$
R=\left\{I|| F_{I q} \mid \geq \tau, I \in I_{T}\right\}
$$

where $F_{I q}$ is the set of features matched between image $I$ and the query image $q, \tau$ is a lower limit of the number of feature matches for $I$ to become the result image, and $I_{T}$ is the set of training images.

There is a trade-off between performance and accuracy in feature matching proce-

dures. As the number of images in the map is large, the set of features in the map is also large. Search timing can be made faster by using approximate nearest neighbor techniques rather than the exact match, but accuracy is affected as more approximation is introduced. To counter this problem, the search space must contain only the set of features which are relevant to the retrieval process, without any duplicates.

The landmark index contains feature descriptors in a data structure that also 
keeps the identification numbers of the frames in which a certain feature is present. Each entry in the structure is given by

$$
\left\langle f_{i},\left\langle c_{i 1}, c_{i 2}, \ldots, c_{i N}\right\rangle\right\rangle, i \in[1, M]
$$

where $M$ is the total number of features, $N$ is the total number of images, $f_{i}$ is the $i^{\text {th }}$ feature descriptor and $c_{i j}, j \in[1, N]$ is a boolean value which is

$$
c_{i j}= \begin{cases}0 & \text { if } f_{i} \text { does not appear in frame } j \\ 1 & \text { if } f_{i} \text { appears in frame } j\end{cases}
$$

With the search space being reduced to a set of features, the image retrieval process is modified to:

First, find the set of indices $X$ of features from training database $F_{T}$ which match with query feature set $F_{Q}$.

$$
X=\left\{x|| \mid f_{x}-f_{q} \|<\epsilon, f_{x} \in F_{T}, f_{q} \in F_{Q}\right\}
$$

where $\epsilon$ is the maximum distance between features to be considered a match. An appropriate $\epsilon$ can be determined empirically with different experiments of feature matching between indoor images.

Then, find the retrieved frame index $k \in[1, N]$ such that

$$
\sum_{x \in X} c_{x k} \text { is maximum and } \geq \delta
$$

where $\delta$ is the pre-set minimum number of matches for retrieval. 


\subsection{Landmarks}

A landmark is defined as a set of distinguished features which are regularly observed and re-observed in the mapping environment. For a feature to be a landmark, it must satisfy a few criteria $[68,72,75]$ :

- A landmark must be stationary. Features from a moving object cannot be a landmark. In an indoor scenario, moving objects, except people, are fewer in number. Moving people generally do not appear in more than one or two frames, so those features can be discarded by filtering.

- A landmark must be distinctive in a map. For example, two similar looking shelves in two sides of a room may lead a system to miscalculate the correct location, and hence they cannot be taken as landmarks. A landmark must be distinctive from its surroundings.

- A landmark must be repeatable. If a region containing a landmark is photographed or visualized from different angles, then the landmark should be present in all of the frames.

\section{- A landmark should be robust against noise and lighting conditions.}

Also, a map should have multiple distinguishable landmarks; otherwise navigation becomes difficult. For this reason, environments with few features, like an urban tunnel, or a hospital floor where all the rooms look very similar, provide a challenge for vision-oriented localization and mapping.

The density of landmarks is another issue in a map. Even if the map has multiple landmarks, it may happen that they are so close together that some of the areas do 
not have any identifiable landmarks. In the proposed algorithm, there is no hard threshold on the number of frames in a map that must contain a landmark, but it is assumed that a sufficient number of images of each area in the indoor environment has at least one distinguishable landmark.

The idea of using features as landmarks was first demonstrated by Se et al. [64] using SIFT features. The landmark identification method described in this thesis follows a similar idea. The map, in this case, consists of multiple overlapping RGB images and corresponding 3D point clouds. The overlap between the images helps to accurately determine a position using multiple frames. For a feature to become a landmark, a number of criteria are imposed in addition to the ones mentioned before.

- A landmark must appear more than once, and in adjacent frames. This ensures that the landmark is repeatable.

- A landmark must not appear in more than a certain number of images. This determines that the landmark is distinguishable, and does not originate from a moving object.

\subsection{Landmark Index Generation}

\subsubsection{Image Acquisition}

Image acquisition is done with a monocular camera. A monochrome camera is sufficient because feature detectors like SIFT work on monochrome images. Each frame is captured in sequence with sufficient overlap with one or more of the previous frames. The overlap region between two frames helps to register the frames to form a single map. There is not a strict rule on how much overlap should exist between two frames. 
In practice, it is found that an overlap of about $30-40 \%$ of image size leads to good performance in the experiments in areas with high feature density, while this may need to increase to $70-80 \%$ for areas with fewer features.

There can be several rounds of collecting images from an environment, because multiple images of the same scene from different angles will not only enrich the collection of features, but also will help to determine which features are re-observed the most number of times. A simple way of performing this task is to first place the camera at the center of a room or hall, rotate the camera $360^{\circ}$ around its vertical axis, and capture all frames. Then move towards the outer edge of the environment, and acquire images around the periphery, which will contain finer features. This further ensures that every visible feature of the entire map is captured in the training images.

The justification of overlapping images in the database, as previously stated, is given by Schindler et al. [62], where they use the concept of information gain. In our method, a feature will be treated as a landmark if it appears in a sufficient number of frames. Overlapping images increases the importance of stable features in this procedure. Stable features will be visible in more sequential frames than unstable ones, increasing the information gain on those features being visible at that location, and thus making them more likely to be chosen as landmarks.

\subsubsection{Implementation}

Figure 3.1 provides a block diagram of the landmark index generation procedure. The landmark index contains SIFT feature descriptors, and the frames in which they appear, in the same data structure described in Section 3.1. In an iterative procedure, from each map image, features $\left\{f_{1}, f_{2}, \ldots\right\}$ are extracted. On the first iteration, all 


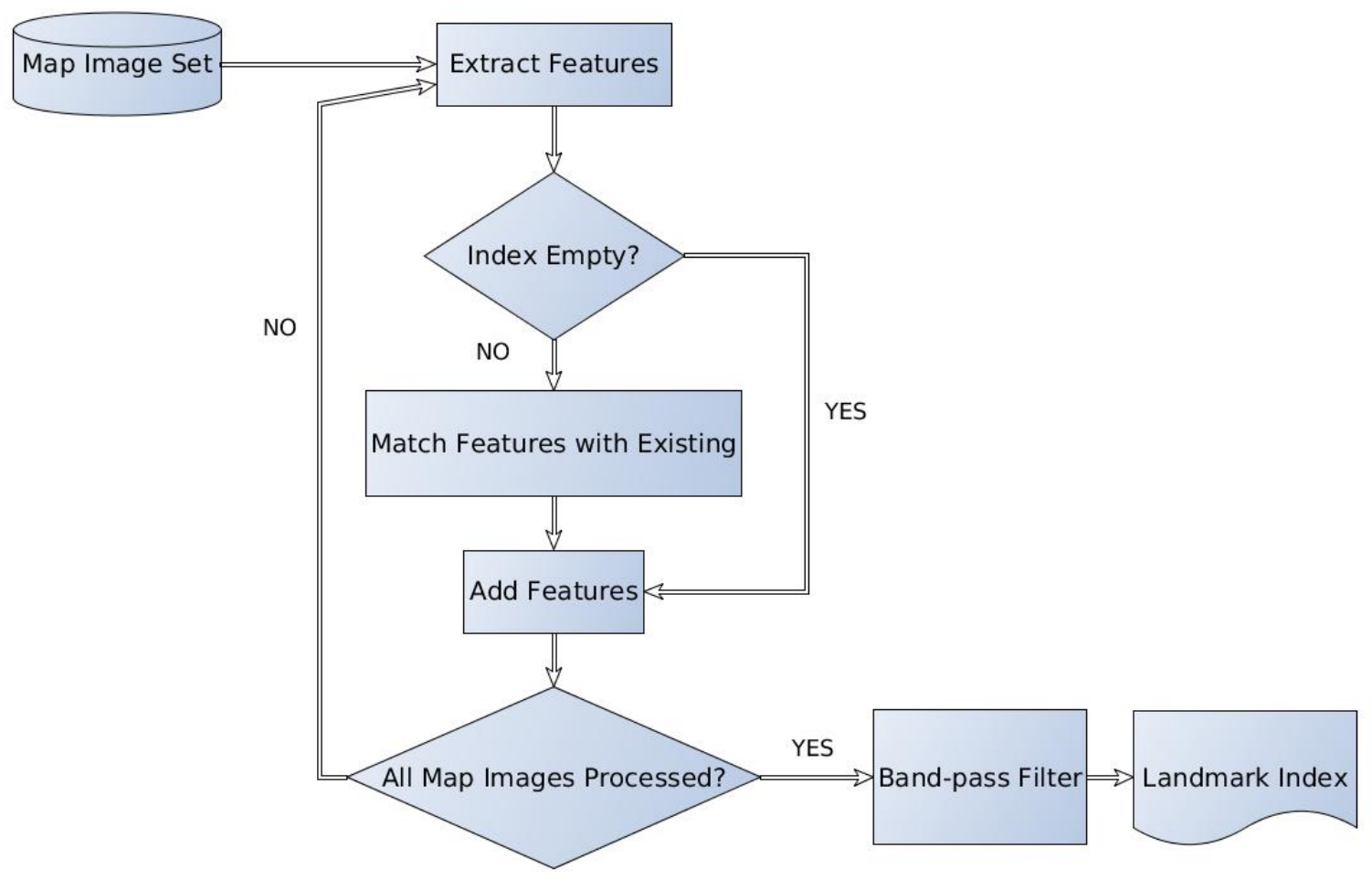

Figure 3.1: Block diagram of landmark index generation procedure

features from the first frame are entered in the index.

$$
f_{i}^{1} \rightarrow l_{i}
$$

where $f_{i}^{1}$ is the $i^{\text {th }}$ feature of the $1^{\text {st }}$ frame and $l_{i}$ is the $i^{\text {th }}$ element in the landmark index.

Subsequently, in each iteration, the extracted features are matched with the existing ones in the index. If a match is found, the existing feature in the index represents both itself and the newly matched feature, given that the distance between the 
matched features is acceptable.

$$
f_{i}^{j} \rightarrow l_{k}=\underset{l_{k}}{\arg \min } \operatorname{dist}\left(f_{i}^{j}, l_{k}\right) \text { s.t. } \operatorname{dist}\left(f_{i}^{j}, l_{k}\right) \leq \tau_{d}
$$

where $\tau_{d}$ is the upper threshold for match distance.

If matches are not found, then the new unmatched feature is simply added to the index. With the addition of each of the matched and unmatched features, the boolean variable $\left(c_{i j}\right)$ associated with frame number is set to 1 .

As stated previously, features must appear in more than one frame and must not appear in all of the frames to be treated as landmarks. Based on this, a postprocessing is applied on the landmark index to filter out the non-landmarks. This post-processing is band-pass filtering, with two thresholds defined on the number of occurrences of the features:

- The lower threshold, $L_{t h}$, represents the minimum number of frames a feature must appear in to be considered as a landmark. This value can be adjusted depending on the image set.

- The upper threshold, $U_{t h}$, is the maximum number of frames in which a feature should occur. If a feature appears in more frames than this number, then it is not considered distinctive. This upper threshold can be a percentage measure on the highest number of times a feature appears in the unprocessed index.

When the processing is complete, the remaining features in the database are considered as landmarks. Algorithm 2 is the complete algorithm to generate the landmark index. 


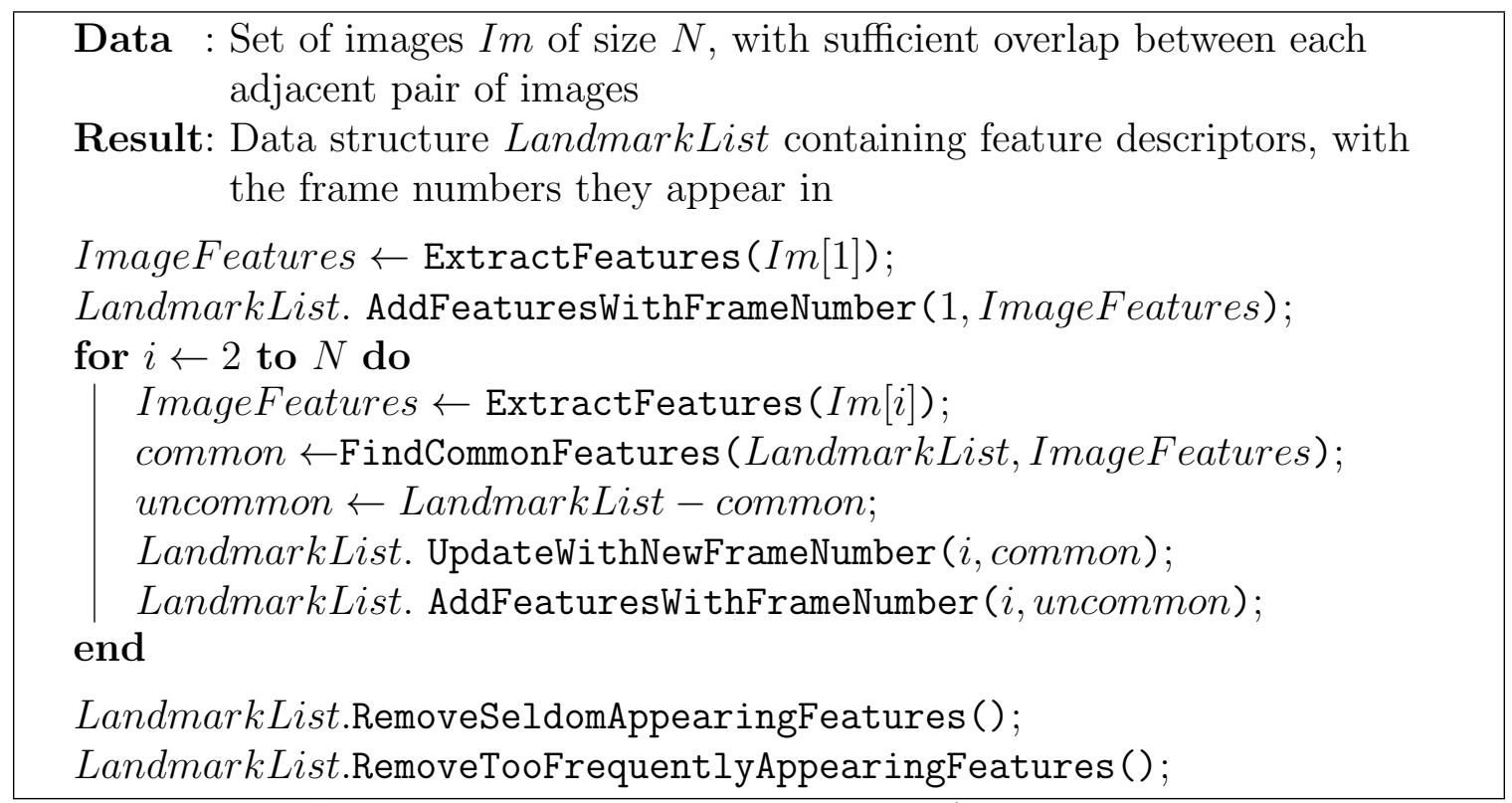

Algorithm 2: Landmark Indexing Algorithm

\subsection{Search Technique}

Figure 3.2 presents a block diagram of the image search procedure. Features are extracted from each query image, and a nearest neighbor search mechanism is employed to match the query features with the landmark index. The Fast Library for Approximate Nearest Neighbor (FLANN) [49] is used for this purpose. The automatic algorithm and parameter configuration of FLANN helps to select the most suitable algorithm between randomized $k$-d trees and hierarchical $k$-means tree and determine the optimal parameters for the selected approach. The optimization process can be geared towards either faster training with slow searching time, or more training time to make the search faster. The second approach is taken here, because the training is done only once during loading the landmark index before searching. The parameters can be saved for reuse of the same map. For each of the feature-to-landmark matches, each frame in the index entry for every matched landmark gets a vote. Once voting 


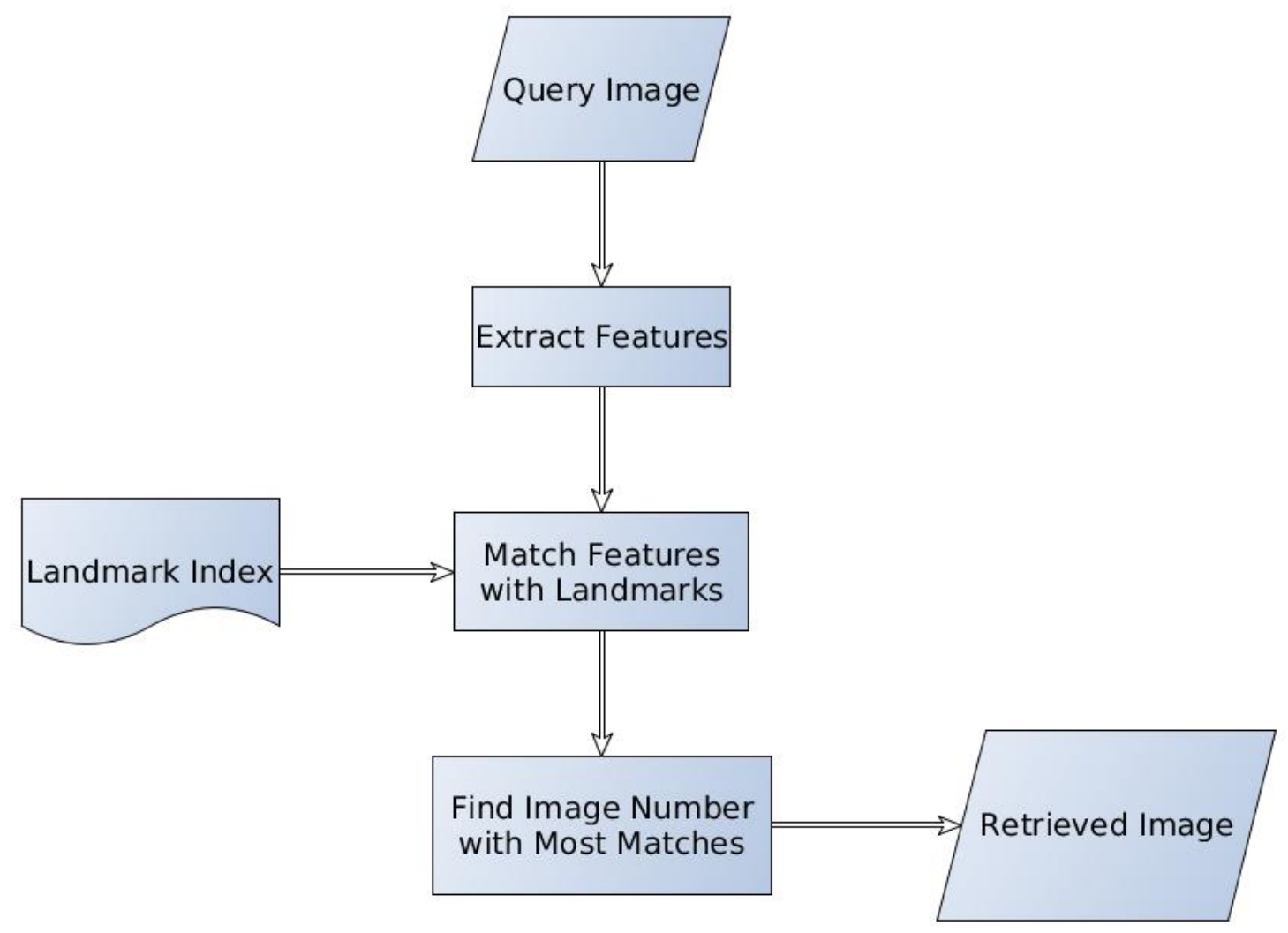

Figure 3.2: Block diagram of image search procedure

is finished, a linear search is performed to find the frame with maximum number of votes. This frame is output as the retrieved image.

From a database containing $N$ training images, a landmark index is built which has $M$ entries, and $k$-d tree is used to find nearest neighbors, then to perform a single query:

- $\mathrm{O}(P \log M)$ time is needed to perform feature matching using $k$-d tree $(\mathrm{O}(\log M)$ time for each feature), where $P$ is the total number of features in the query image;

- $\mathrm{O}(P N)$ time is needed to use linear search on the frame number list, and vote 
for all frames for each of the $P$ matches;

- $\mathrm{O}(N)$ time is required to perform another linear search on the frame number list to find the frame with maximum vote.

It can be recalled from previous discussion (Section 2.2) that, bag of visual words takes:

- $\mathrm{O}(M \log P)$ time to build the term frequency histogram for the query image;

- $\mathrm{O}(M N)$ time to find the scalar product of the query histogram with those from the $N$ training images, and find the largest scalar product.

Considering $M>>P$, the total time complexity of image retrieval using landmark indexing, $\mathrm{O}(P N)$ is much less than that using bag of visual words, $\mathrm{O}(M N)$.

Image retrieval depending on feature matches only may result in false positives because of outliers that do not satisfy the epipolar constraints between two images. To correct this problem, the fundamental matrix is estimated from the feature correspondences. If there are at least 8 correspondences, a RANSAC approach is applied, and outliers are removed which do not fall inside the inlier threshold. If the retrieved image, which has the maximum vote using the landmark index, does not contain enough matches after filtering out the incorrect correspondences, then that match is rejected, and the candidate with the second highest number of votes is considered. This goes on until an image is found which satisfies the epipolar constraint, or the remaining candidates are found to have votes $<\delta$ (the previously defined minimum number of matches for acceptable retrieval), in which case no suitable match is retrieved for the query image. 


\subsection{Demonstration in 3D Navigation}

The image retrieval system using landmark indexing can be demonstrated in an indoor navigation application. A brief overview of the navigation system is described here. The system has two phases: pre-processing (mapping) and runtime (navigation).

\subsubsection{Pre-processing}

The pre-processing phase is mainly the map generation and storage procedure. A $\boldsymbol{m a p}$ is a collection of 2D images and corresponding 3D point clouds, organized in a data structure with the registration information. For the runtime navigation phase to work, all components of the map should be properly stored. This phase is performed with a Kinect camera and a modified RGBDSLAM [17] interface. As suggested in Section 3.3.1, to create the map, the recommended procedure is to position the camera at the center of the room, rotate $360^{\circ}$ and capture all frames for processing. Once this is complete, the camera should be moved towards corners and far ends of the room to capture detailed frames with close up shots. This helps to identify landmarks from any distance during navigation. Also, the frames must overlap to identify the common features as potential landmarks.

For the purpose of 3D registration error minimization only, RGBDSLAM uses a graph optimization algorithm, which is discussed later. This graph representation is not related to the image retrieval procedure.

The framework for pre-processing phase is given in Figure 3.3. 


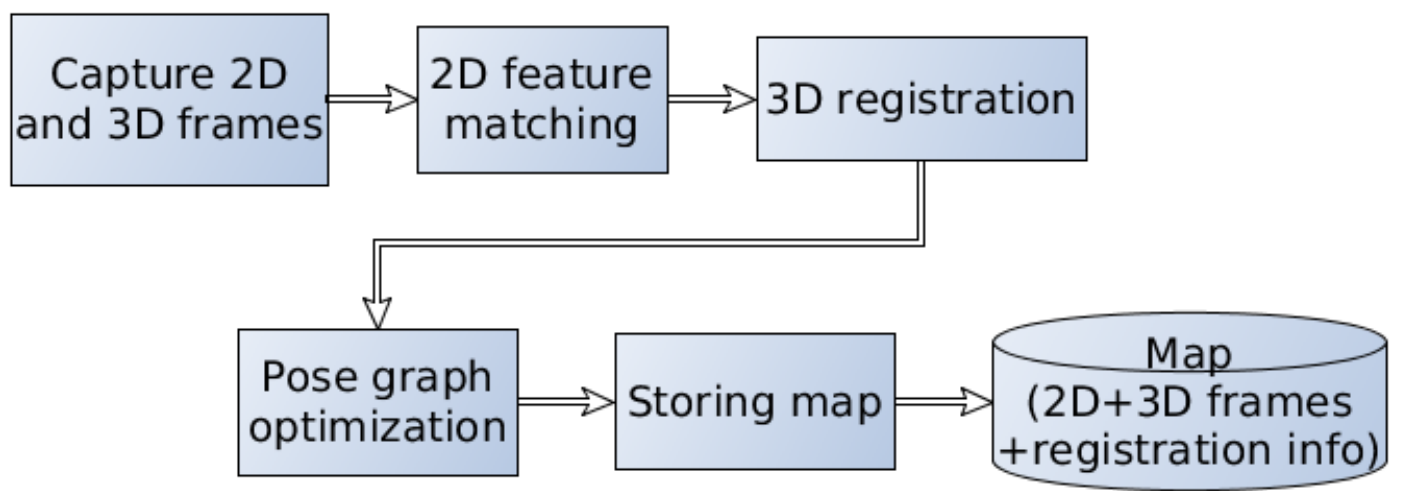

Figure 3.3: Block diagram of pre-processing

\section{Feature-based Matching}

Both 2D and 3D frames are captured with the help of the Kinect camera. From the 2D RGB frames, features are extracted. To be consistent with the rest of the work, only SIFT features are used here.

When each frame is captured, it is matched with the pre-existing frames. This is performed with Fast Library for Approximate Nearest Matching (FLANN). The correspondences found between two images are filtered in two steps.

1. The matching is performed twice. The query and training images are swapped and re-matched. Common correspondences from both rounds are considered as correct.

2. From the correspondences, the fundamental matrix between the two images is computed. This matrix is applied on the query image to match with the target, and the re-projection error is estimated. The match is considered accurate if the error lies below a threshold.

These steps may take some time. However, the pre-processing phase does not need 
to be fast; it only needs to be accurate so that the navigation phase can be real-time.

\section{D Pose Determination using RANSAC}

This is done using the same procedure described in the Section 2.3.1.

The 2D feature correspondences found in the previous step are transformed into 3D point correspondences between the point clouds. This is possible because the point clouds are organized in a $640 \times 480$ pixel grid, which means all 3D points can be addressed with 2D image coordinates. The RANSAC algorithm (Algorithm 1) estimates the pose of each frame from these correspondences.

\section{Graph Representation of Map}

In a graph representation $G=(V, E)$, each vertex/node $v \in V$ contains a frame of the map:

$$
v=\left(I_{i}, I_{d}, F_{i}, C, P_{c}\right)
$$

where $I_{i}$ is the intensity image, $I_{d}$ is the depth image, $F_{i}$ is the set of image features (both interest points and feature descriptors), $C$ is the 3D point cloud and $P_{c}$ is the set of 3D interest points. Additional information may be added if required.

Each edge $e \in E$ between two vertices $v_{1}$ and $v_{2}$ contains match information between them:

$$
e_{v_{1}, v_{2}}=\left(X_{1}, X_{2}, t, w\right)
$$

where $X_{1}$ and $X_{2}$ are the lists of indexes of points in frames of $v_{1}$ and $v_{2}$ respectively, and $t$ is the transformation matrix of $v_{2}$ with respect to $v_{1}$, and $w$ is a weight assigned to the edge. The number of feature correspondences between two frames can be used as the weight on the edge, because fewer correspondences most probably 
means a wrong frame match, and more correspondences most probably means better frame-to-frame registration. This weight is used in traversal and optimization of the graph structure. Further emphasis on the weight can be applied by using a higher exponentiation on the count of correspondences.

The graph optimization algorithm used here is $\mathrm{g}^{2} \mathrm{o}$ (for general graph optimization) [37]. It is a generalized, least squares optimization technique of an error function represented in a graph. This optimization procedure is very useful in loop closure detection, which is the identification of revisiting previously explored locations. Any accumulated error can be minimized during loop closure by revisiting old locations, and propagated throughout the graph to correct the entire 3D model.

\section{Map Saving}

The final step of the pre-processing phase incorporates saving the RGB image of each frame, as well as the 3D point cloud, and the transformation matrix with respect to the first frame. The first frame is taken as the base frame and has a identity matrix as its transformation.

\subsubsection{Runtime}

The steps performed in the runtime or navigation phase are not entirely different than pre-processing, except that the landmark indexing technique is used to retrieve images and localize in real time. The 3D pose calculation is the same as before. The procedure is depicted in a flowchart in Figure 3.4. 


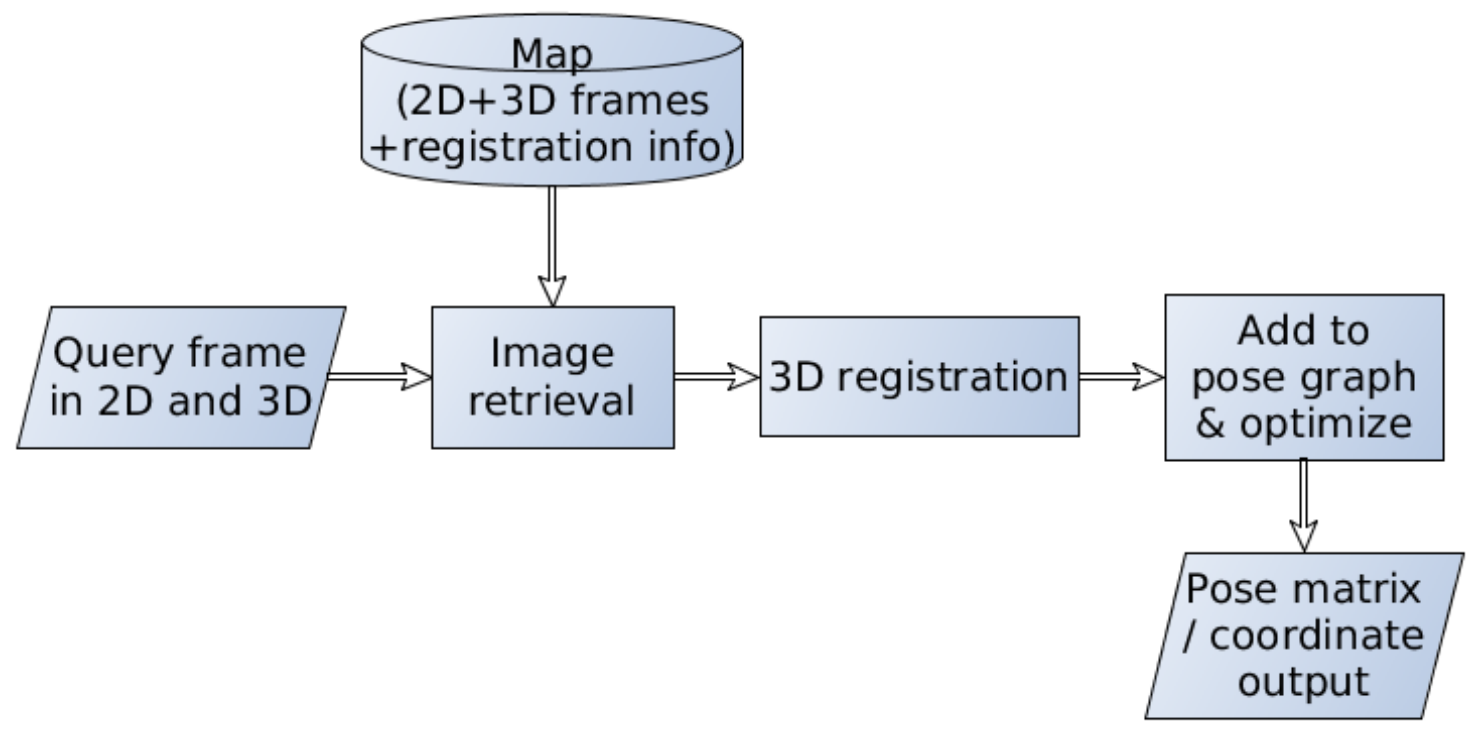

Figure 3.4: Block diagram of runtime

\section{Application of Landmark Indexing}

The graph traversal method of RGBDSLAM is replaced here with the feature-based landmark indexing. Each image of the map is passed through Algorithm 2 to generate the index. This can be done during loading time of the map into the navigation system, or can be processed at the map generation phase and saved in a file during the pre-processing phase.

In runtime, the Kinect camera captures frames and goes through the image retrieval procedure. This procedure can be modified to present multiple output frames instead of one, so that, if the captured frame is put as a node in the graph representation of the map, it can have multiple edges with the map nodes. In this case, the most probable map frames that match current captured frame are the neighbors of the best matched image. More than one edge for a node also helps in the optimization process of the graph. There may be outliers, but those can be detected with a threshold on 
the number of correspondences found in the matching process, because any outlier should have a significant low number of matches. Further filtering can be performed with the previously discussed epipolar constraint verification.

\section{Pose Determination and Addition to Graph}

Once the image is retrieved, The previous 3D pose determination step is followed. Then, the frame is added to the existing map graph as a node, and an edge with the correspondence count and transformation matrix is added. If multiple images are retrieved, one edge is added for each of them. Then the $\mathrm{g}^{2} \mathrm{O}$ optimization procedure is applied over the graph.

Adding nodes in graph makes the system remember the previous location and orientation. If there is any such previous memory, which will occur after the first node is added in runtime, then an edge can be added with this previous node. This improves localization. However, if the camera is moving very fast, then there may be a large rotation or translation factor between those two frames, and this edge can be neglected. The maximum allowable movement speed depends on the processing time of the algorithm. Generally, from practical experience on RGBDSLAM, it is normal human walking speed, which is approximately $1.5 \mathrm{~m} / \mathrm{s}$. Also, if the camera is not moving at all, then the transformation matrix will be close to an identity matrix. This condition can be used to abort the unnecessary registration procedure.

\section{Error Avoidance and Correction}

The $\mathrm{g}^{2} \mathrm{O}$ optimization helps to minimize error between the added frame and map. However, the chance of accumulation of error is never diminished. The result will 
be similar to that when a GPS receiver fails to locate itself and shows the user a completely different street address. So, as a fail-safe, a certain maximum number of nodes can be remembered by the system. After exceeding this limit, the system clears all the navigation data except the map itself, and relocates itself using image retrieval. 


\section{Chapter 4}

\section{Experimental Results}

Experiments were performed to retrieve location images from different image datasets. These datasets were either downloaded from online sources or captured manually, and in both cases the proposed landmark indexing algorithm performed better than the method of Liang et al. [39] or the bag of visual words [66]. The method was further applied to localize a user in a 3D map in real time, as described in the previous chapter.

\subsection{Data Acquisition}

The image retrieval method for landmark indexing was tested on a few different datasets. Three of these datasets consisted of images acquired by the author, one was an indoor navigation dataset available online, and the third was created from recorded videos in an underground mine tunnel.

Manually acquired sets. These datasets consisted of frames extracted from videos captured in the following areas:

1. Queen's University Electrical \& Computer Engineering (ECE) Department 
office;

2. A laboratory room;

3. Partial 1st and 2nd floors of Cataraqui Shopping Center, Kingston.

The videos were captured with the 8 megapixel camera of a Samsung Galaxy S4 phone. All extracted frames were downscaled to $640 \times 480$. They had considerable overlap, which can be seen in a partial shopping center dataset shown in Figure 4.1. Some images were separately captured at random locations in these areas and used as query. 

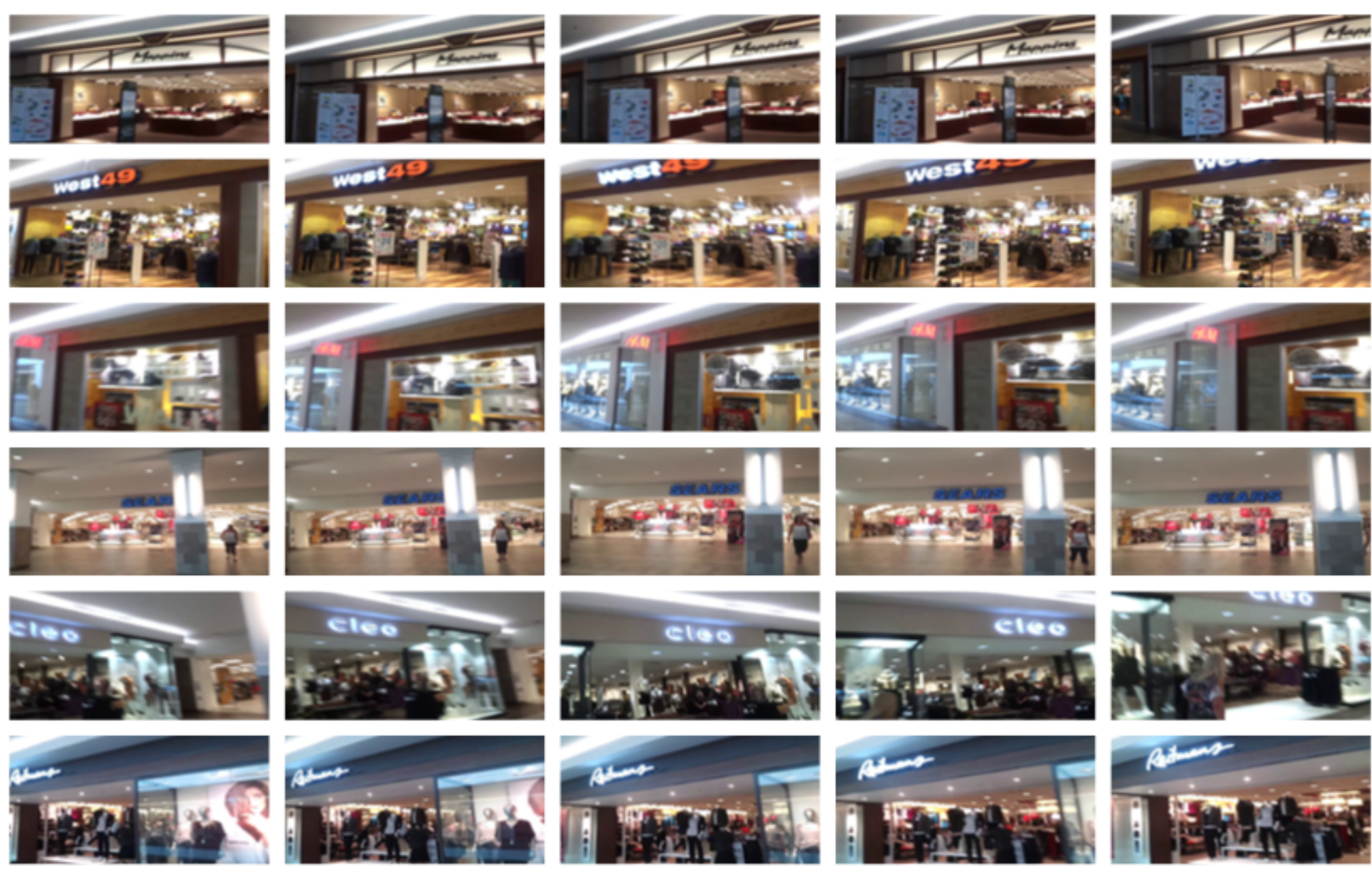

Figure 4.1: Partial shopping mall dataset which shows the overlapping training images. 
Online dataset. The Cognitive Navigation [36] dataset consists of $640 \times 480$ images of different indoor areas (a corridor, an office, a laboratory, an elevator area and a bathroom). It is a three-part dataset, where the first part contains images of individual areas separately in natural illumination conditions, the second part is the same but captured at night with artificial lighting, and the third part is a continuous exploration of all the places in artificial illumination conditions. The images are captured with a Microsoft Kinect. For the experiment, the 3rd part was chosen, because it had more than 500 images with sufficient overlap, from which nearly $450 \mathrm{k}$ features could be extracted. Due to the absence of separate query images, some random images were chosen as queries and were not included during training.

Underground mine tunnel. Two high-quality videos captured with a video camera mounted on a mine vehicle were used for experimenting. Both videos showed the same tunnel, the only difference being the driving direction. The first video captured the tunnel as the vehicle moved forward. In the second video, the vehicle was on reverse gear. Focused lighting from the headlights of the vehicle and occasionally present overhead lights were the sources of illumination in the tunnel. 598 frames extracted from the first video were used to build the landmark index, and 65 images extracted from the second video were used as query. All images were downscaled to $640 \times 480$.

For the demonstration of the landmark indexing in indoor navigation, 3D point clouds were collected in the ECE Department office, and GeoDigital International lobby, with a Microsoft Kinect. All frames were $640 \times 480$ organized point clouds. 


\subsection{Filter Parameter Estimation}

To estimate the best parameter for the bandpass filter, image retrieval experiments were performed using different values of the lower and upper thresholds, and it was estimated which values provide the shortest length feature index with maximum retrieval accuracy. The result of this experiment was a pair of the highest possible lower threshold and the lowest possible upper threshold $\left(L_{t h}, U_{t h}\right)$ for each dataset. To find this, the following were measured:

- True Positive Rate $(T P R)$ or Recall $=\frac{T P}{T P+F N}$

- False Positive Rate $(F P R)=\frac{F P}{F P+T N}$

- Accuracy $=\frac{T P+T N}{T P+F P+T N+F N}$

where $T P=$ number of true positives, $T N=$ number of true negatives, $F P=$ number of false positives, and $F N=$ number of false negatives. Each pair of $\left(L_{t h}, U_{t h}\right)$ thresholds generated a single $(F P R, T P R)$ point in a Receiver Operator Characteristic $(\mathrm{ROC})$ space. In this space, the point closest to $(0,1)$ showed the best parameters. However, multiple threshold values might generate the same TPR and FPR. To choose the best parameters, the closest point to $(0,1)$ in ROC space was selected, and then from all of the corresponding pairs $\left(L_{t h}, U_{t h}\right)$, the one which created the smallest set of landmarks and showed the highest accuracy was chosen. For the lab dataset, the pair $(12,80 \%)$ gives the lowest FPR, with a sufficiently high TPR and the highest accuracy. So, $(12,80 \%)$ are the optimal parameters for the lab dataset. Parameters for all datasets are listed in Table 4.1. An example ROC plot for the Laboratory dataset is shown in Figure 4.2. 


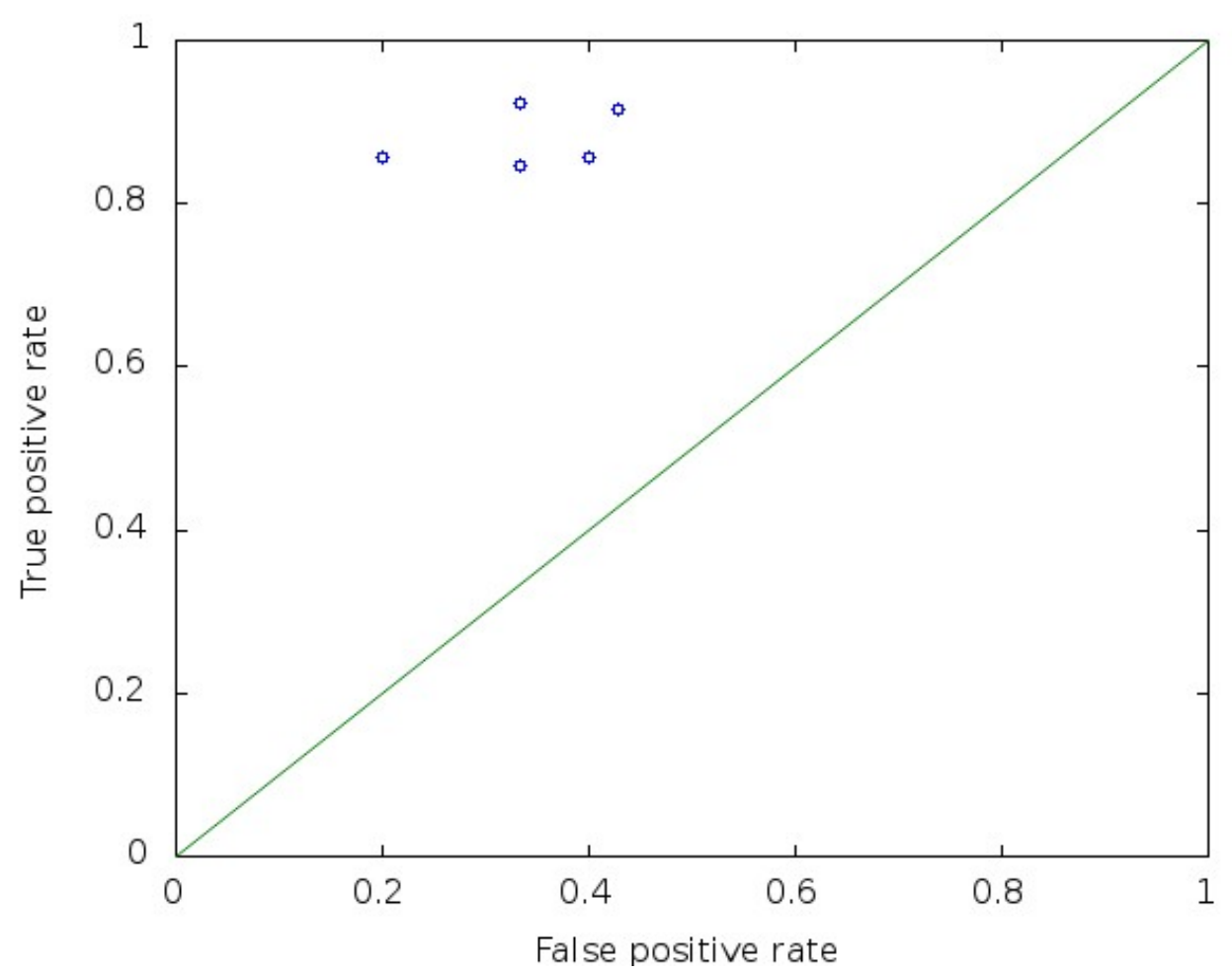

Figure 4.2: ROC plot for different lower and upper thresholds of the bandpass filter applied on the laboratory dataset. Multiple threshold pairs can generate same (FPR,TPR) point. The threshold pair that generates a point closest to $(0,1)$ in ROC space and creates the landmark index with smallest size is chosen for the particular dataset.

Table 4.1: Optimal filter parameters for different datasets

\begin{tabular}{|c|c|}
\hline Dataset Name & Optimal $\left(L_{t h}, U_{t h}\right)$ \\
\hline Office & $(6,80 \%)$ \\
\hline Lab & $(12,80 \%)$ \\
\hline Cataraqui Mall & $(6,80 \%)$ \\
\hline Cognitive Navigation & $(14,50 \%)$ \\
\hline Mine tunnel & $(0,100 \%)$ \\
\hline
\end{tabular}


The above table shows that none of the features were removed from the landmark index for the mine dataset. The reason for this was the presence of a very low number of features in the environment, so that any removal would increase the error.

\subsection{Landmark Index Generation Results}

Table 4.2 presents the size of each landmark index after applying the optimal parameters for each dataset. The Turcot-Lowe method [72], as described in Section 2.2, reduces the feature set to about $4 \%$ of the complete collection using image adjacency graphs. In the described experiments, it was seen that the landmark index contained $14-18 \%$ of all features. The reason is that while [72] uses random outdoor images of buildings in a city, the proposed method works with indoor environments. In an outdoor image, due to the presence of people and moving vehicles, the features on the buildings and stationary roadside objects are fewer. In an indoor setting, chairs, tables, decorations on the wall, poster, signboards etc. provide a very large set of landmarks. In our experiments, it was found that reducing the feature set below this optimal level started introducing errors in image retrieval. 
Table 4.2: Landmark Index Generation Results

\begin{tabular}{|c|c|c|c|}
\hline $\begin{array}{l}\text { Dataset } \\
\text { Name }\end{array}$ & $\begin{array}{l}\text { Total \# } \\
\text { of Frames }\end{array}$ & $\begin{array}{l}\text { Total \# of } \\
\text { Features from } \\
\text { Frames }\end{array}$ & $\begin{array}{l}\text { \# of Landmarks } \\
\text { in Database }\end{array}$ \\
\hline Office & 176 & 43886 & $7837(17.86 \%)$ \\
\hline Lab & 220 & 127558 & $20392(15.98 \%)$ \\
\hline Mall $1^{\text {st }} \mathrm{Fl}$ & 255 & 232829 & $41545(17.84 \%)$ \\
\hline Mall $2^{\text {nd }} \mathrm{Fl}$. & 285 & 251260 & $43430(17.28 \%)$ \\
\hline $\begin{array}{l}\text { Cognitive } \\
\text { Navigation }\end{array}$ & 532 & 456221 & $62304(13.66 \%)$ \\
\hline Mine tunnel & 598 & 141285 & $91117(64.49 \%)$ \\
\hline
\end{tabular}

The underground mine is a special scenario regarding presence of features. 598 frames were extracted from the training video, and only 141285 features were extracted from these frames. In comparison,the indoor environment from Cognitive Navigation dataset with more than 500 frames could produce more than $450 \mathrm{k}$ features. Initial experiment with this dataset showed that using the feature matching resulted in generating a landmark index containing only 23715 features, and image retrieval experiments using this index showed a very low (40.32\%) accuracy.

To solve this problem, the first approach taken was to incorporate all 141285 features in the landmark index. Searching using this set of landmarks produced $100 \%$ accuracy.

The second solution was to use an upper threshold for match distance to filter the 
feature matches. Because most of the features in mine were very similar, simply finding the nearest neighbor did not consider the subtle differences between two similar feature descriptors. This distance was measured in 128-D feature descriptor space. A threshold of 150.0 units was found optimal, which generated a landmark index with $100 \%$ search accuracy.

\subsection{Search Accuracy and Performance}

Table 4.2 presents the accuracy and performance of searching using landmark index for each dataset. The performance was measured by taking the average of search time for all queries. Figure 4.3 shows how the database image with the maximum landmark match count is chosen as the retrieved image. The landmark match count histogram for each example in Figure 4.3 depicts the number of query-feature-tolandmark matches, i.e., $\sum_{x \in X} c_{x k}, k \in[1, N]$ as described in Section 3.1, for all the training images. Figure 4.4 shows some examples of incorrect retrieval. Figure 4.5 shows a comparison of the accuracy and performance of our landmark indexing method with those of the exhaustive feature set method of Liang et al. [39], and bag of visual words [66]. 
Table 4.3: Search Accuracy and Performance

\begin{tabular}{|c|c|c|c|}
\hline $\begin{array}{l}\text { Dataset } \\
\text { Name }\end{array}$ & $\begin{array}{l}\# \quad \text { of } \\
\text { Query } \\
\text { Images }\end{array}$ & $\begin{array}{l}\text { Accuracy with } \\
\text { Landmark In- } \\
\text { dexing }\end{array}$ & $\begin{array}{l}\text { Average } \\
\text { Search Time }\end{array}$ \\
\hline Office & 38 & $100 \%(38)$ & $10 \mathrm{~ms}$ \\
\hline Lab & 47 & $95.74 \%(45)$ & $15 \mathrm{~ms}$ \\
\hline Mall $1^{\text {st }} \mathrm{Fl}$ & 55 & $90.91 \%(50)$ & $30 \mathrm{~ms}$ \\
\hline Mall $2^{\text {nd }} \mathrm{Fl}$ & 60 & $93.33 \%(56)$ & $28 \mathrm{~ms}$ \\
\hline $\begin{array}{l}\text { Cognitive } \\
\text { Navigation }\end{array}$ & 60 & $100 \%(60)$ & $20 \mathrm{~ms}$ \\
\hline Mine tunnel & 62 & $100 \%(62)$ & $20 \mathrm{~ms}$ \\
\hline
\end{tabular}

It can be observed that the landmark match count histograms for false positives were much flatter than those for true positives. The reason was the existence of query features which were very common to the environment. For example, Figure 4.4a shows a query image, which has computers and tables that are common to the rest of the lab area, so feature-to-landmark match counts are almost evenly distributed for the training images. Similarly in Figure 4.4b features from mall windows and mannequins cause false positive retrieval. Determining the nature of histograms can be used to predict false positives in future developments of the search algorithm.

From Figure $4.5 \mathrm{~b}$ it can be observed that the average time consumption for image retrieval using Liang et al. is much less than reported (2-4 seconds) in [39]. However, with increasing size of image dataset, the feature collection will also increase, and the performance will be significantly affected. Their exhaustive feature set will increase 
linearly with addition of each image in the dataset, but it will be much less, possibly logarithmic, in landmark indexing because of internal feature matching and removal of useless features. This can be verified in future work. 

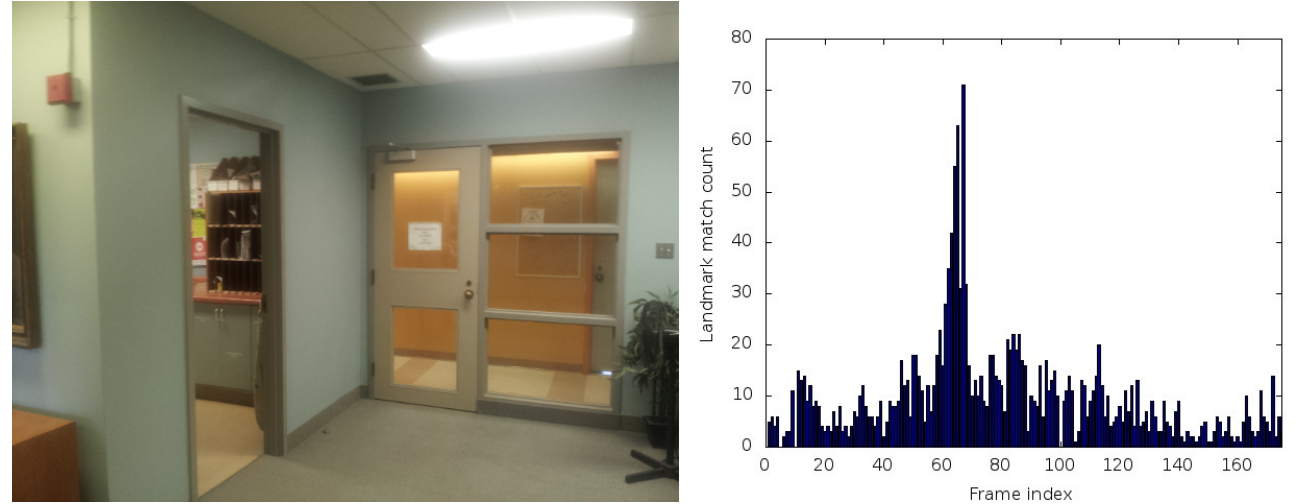

(a) Office
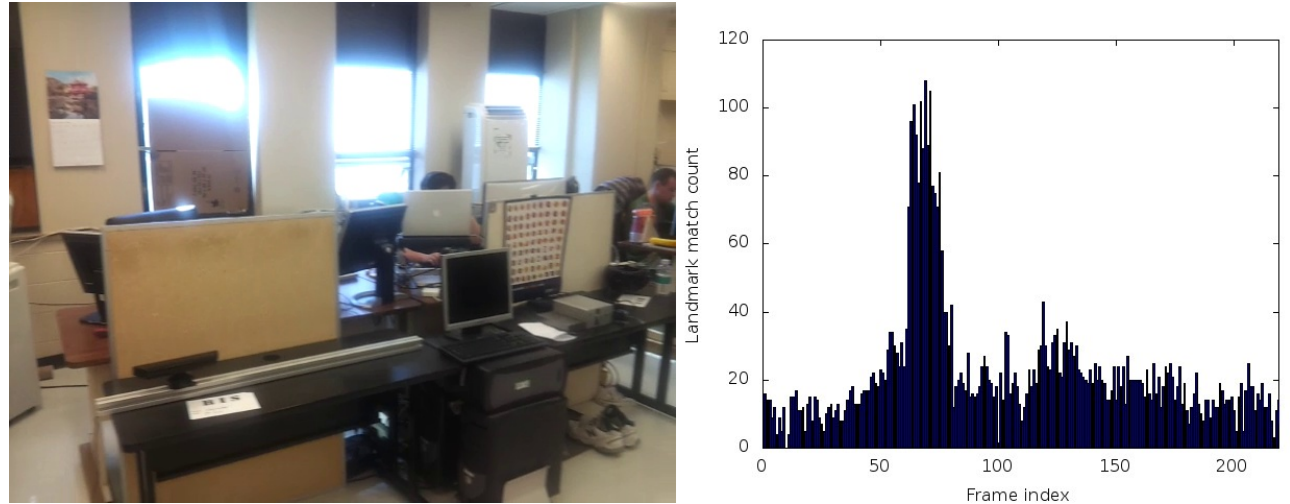

(b) Lab
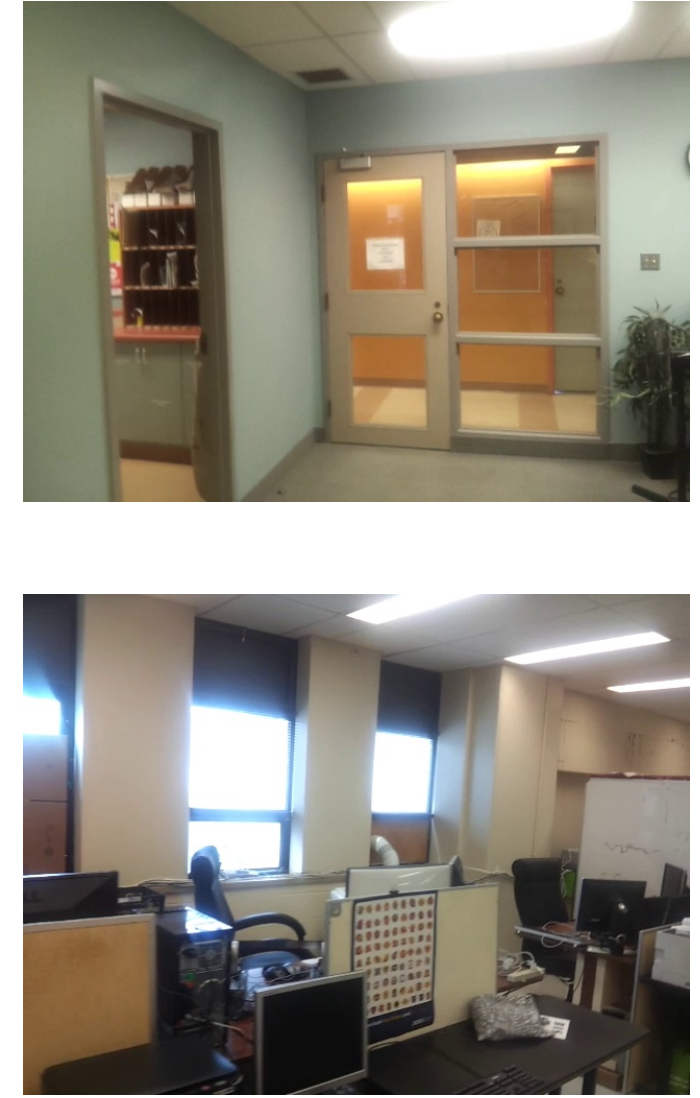

Figure 4.3: True Positive image retrieval results using landmark indexing. left) Query image, center) Landmark match count histogram between the query and each database image, right) Matched image from training dataset, which corresponds to the peak in the histogram. 

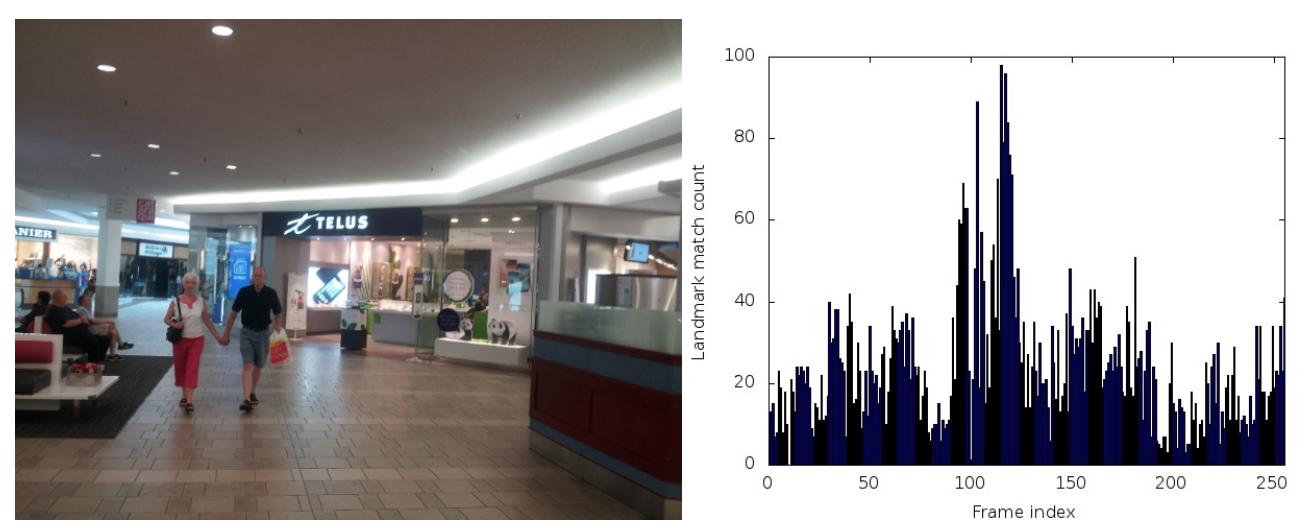

(c) Shopping mall
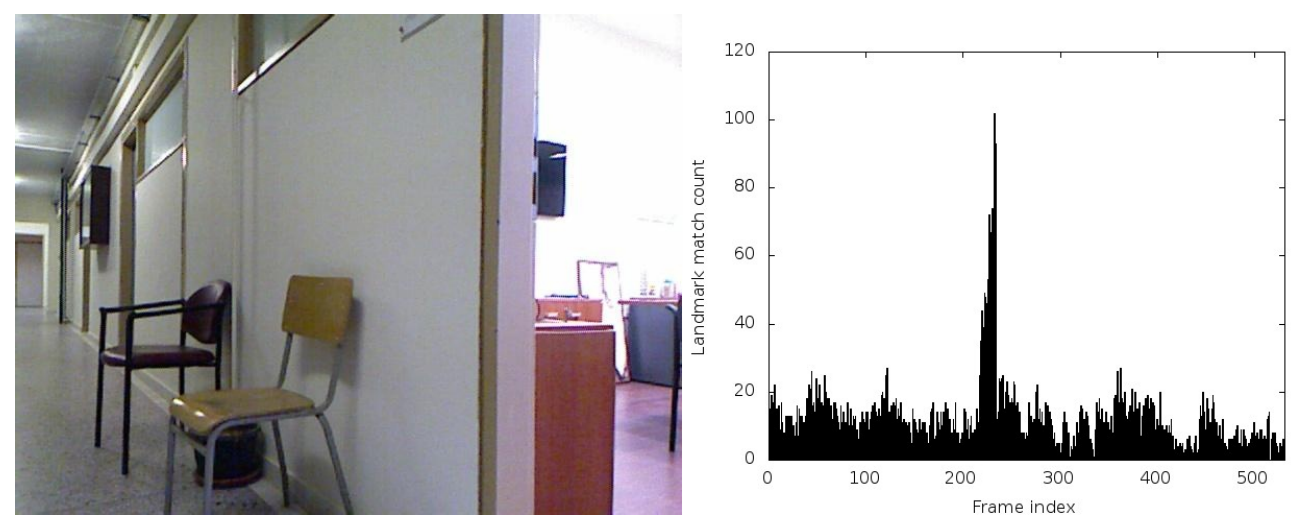

(d) Cognitive Navigation dataset

Figure 4.3: (Continued) True Positive image retrieval results using landmark indexing. left) Query image, center) Landmark match count histogram between the query and each database image, right) Matched image from training dataset, which corresponds to the peak in the histogram. 

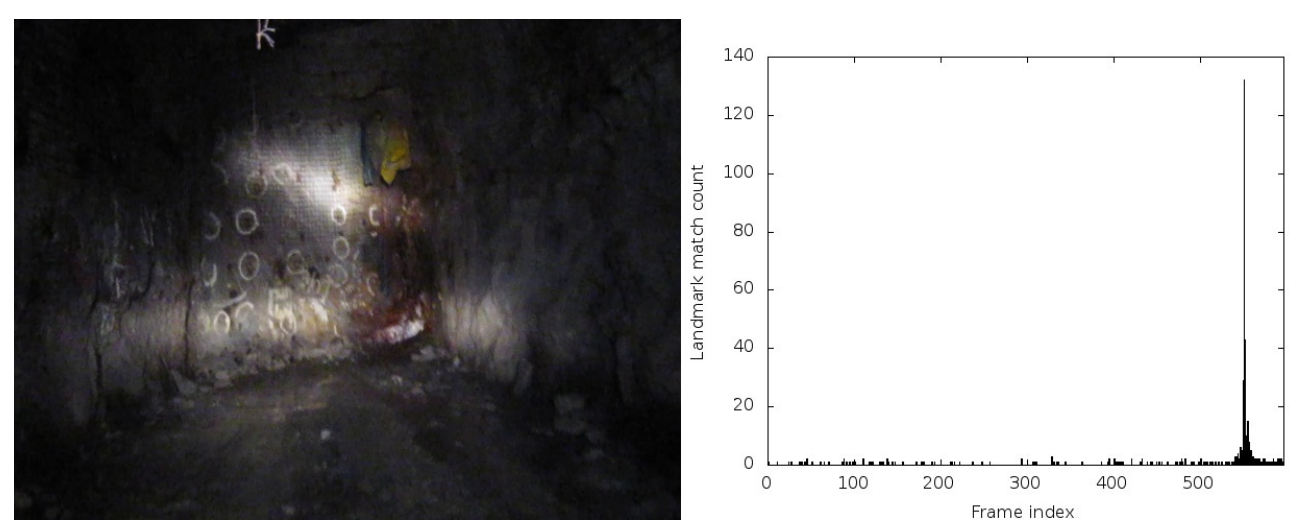

(e) Underground mine wall
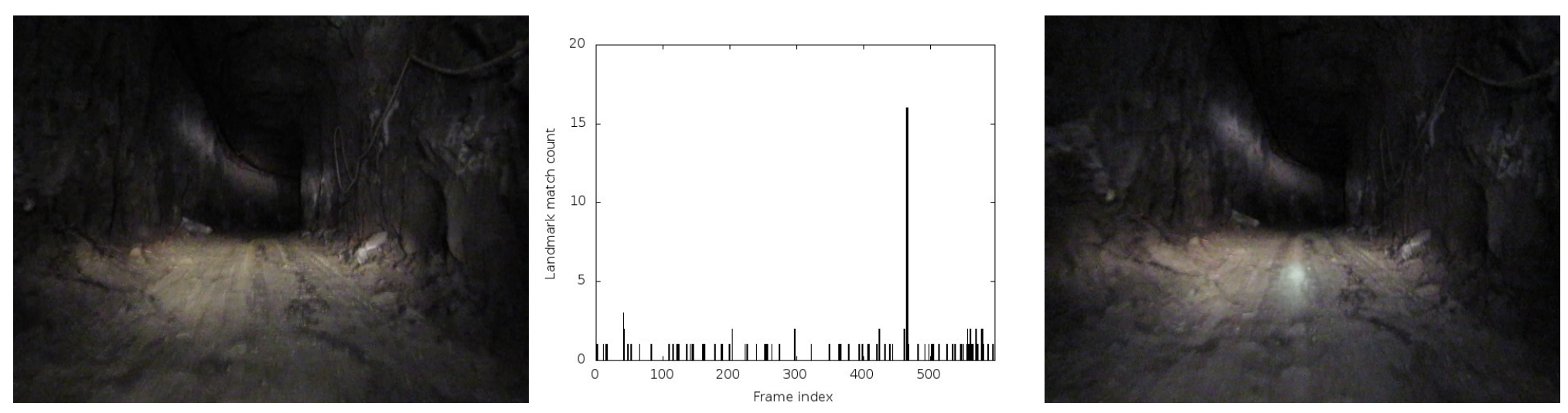

(f) Underground mine tunnel

Figure 4.3: (Continued) True Positive image retrieval results using landmark indexing. left) Query image, center) Landmark match count histogram between the query and each database image, right) Matched image from training dataset, which corresponds to the peak in the histogram. 

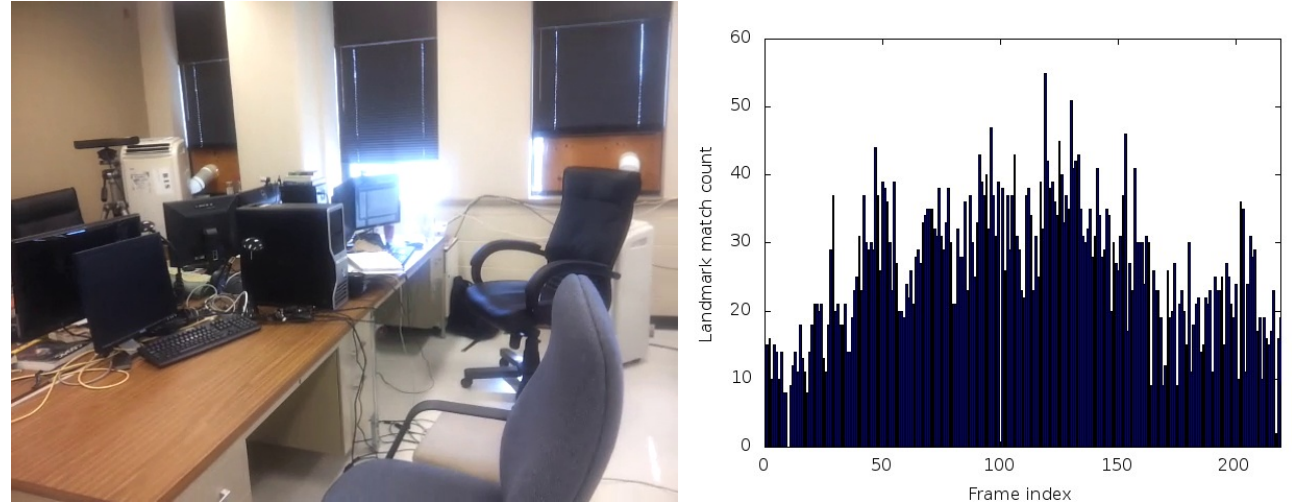

(a) Lab
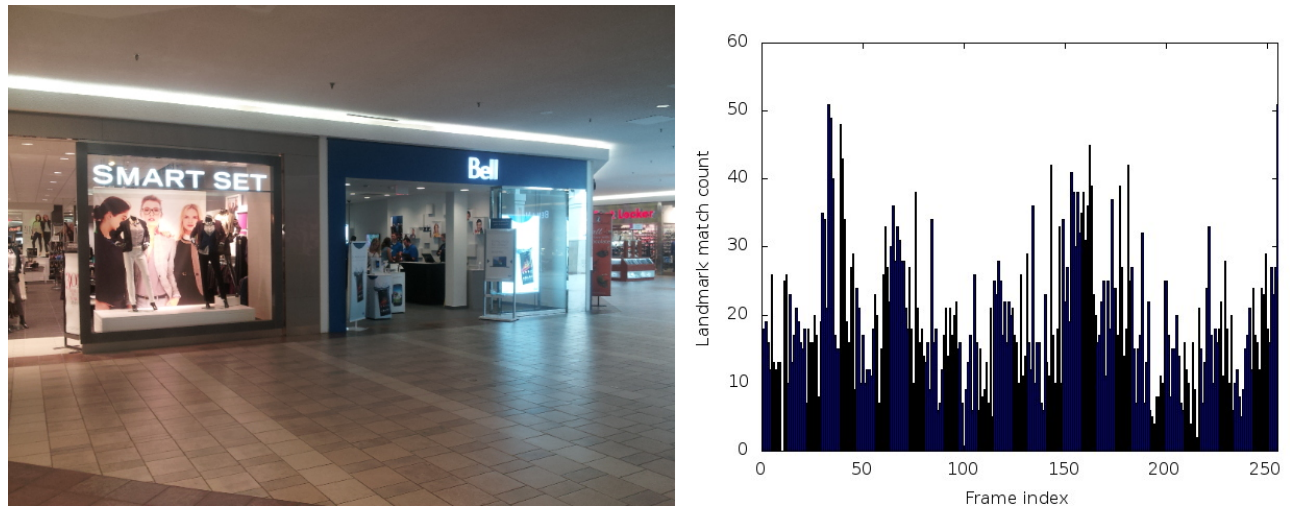

(b) Shopping mall

Figure 4.4: False Positive image retrieval results using landmark indexing. left) Query image, center) Landmark match count histogram between the query and each database image, right) Matched image from training dataset. 


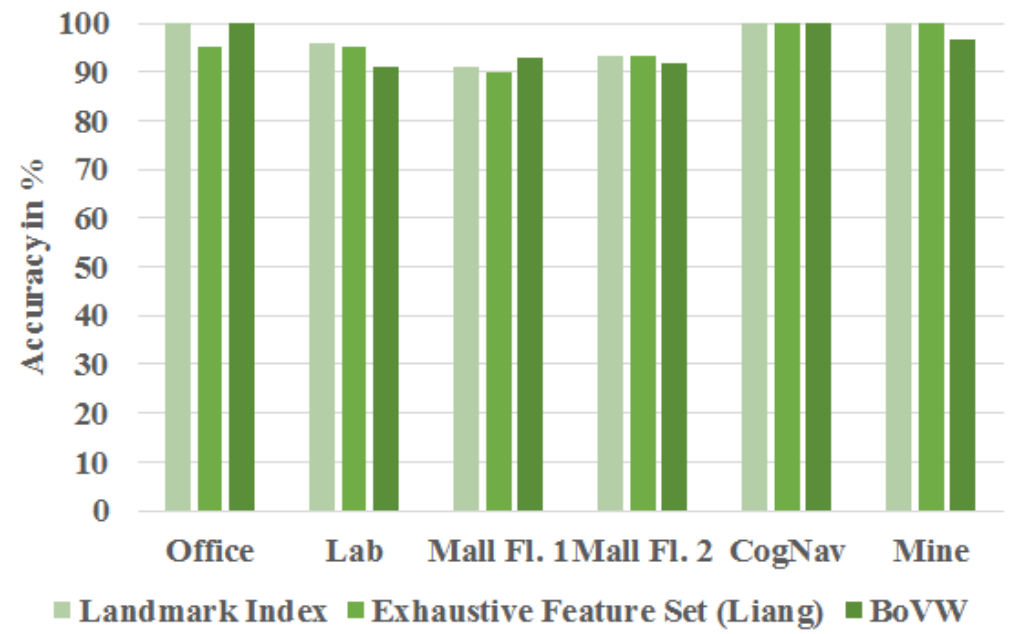

(a) Accuracy Comparison

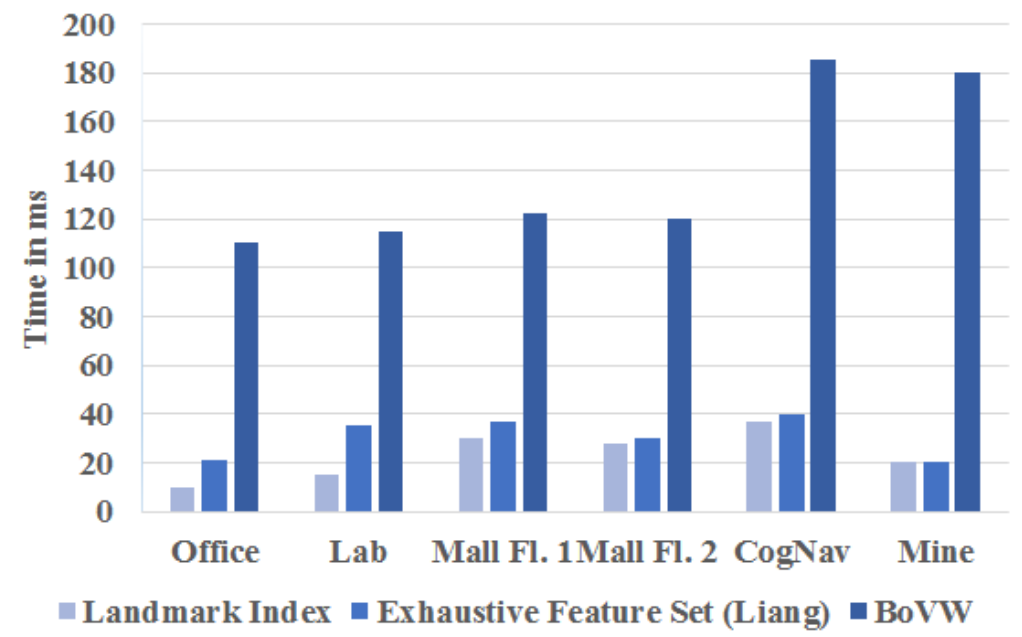

(b) Performance Comparison

Figure 4.5: Image retrieval accuracy and performance comparison between our method, the exhaustive feature set approach of Liang et al. and bag of visual words. All methods show comparable accuracy, however, the time performance of our Landmark Indexing method is much better than bag of visual words. 


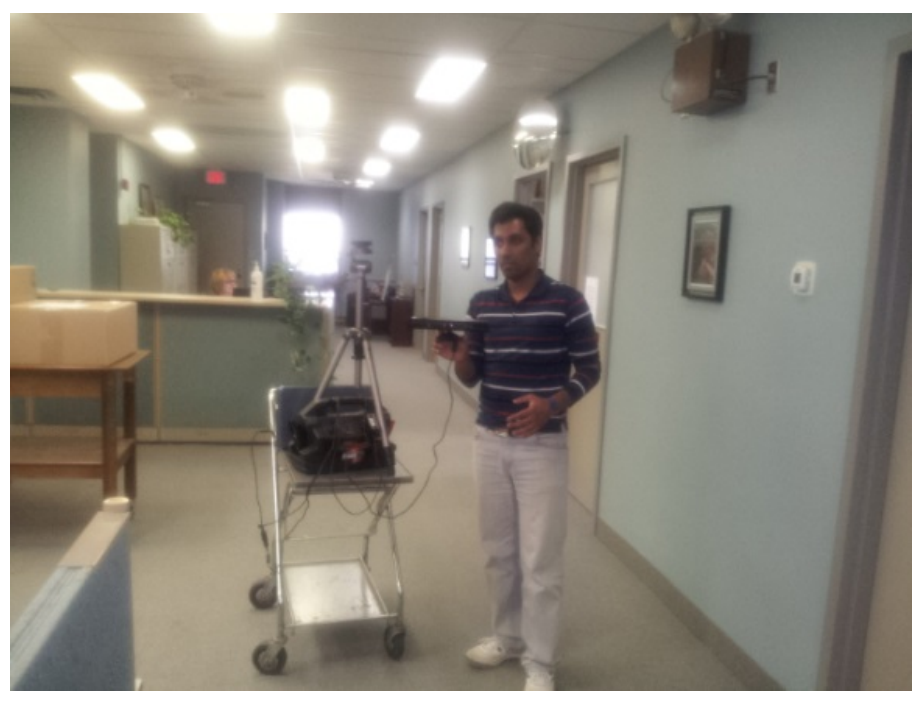

Figure 4.6: The Indoor Positioning System Setup

\subsection{Indoor Navigation}

3D maps for this experiment were constructed using the Microsoft Kinect camera, and the modified RGBDSLAM interface. The indoor navigation system was designed by mounting the camera on a tripod, and then keeping the tripod, the controlling laptop, and a portable power source on a hand-cart. The tripod was adjusted to maintain the Kinect at eye level. The system is pictured in Figure 4.6. 3D map building was not of concern because the goal was to demonstrate the landmark indexing method in a practical application. It was manually verified that the map generation process was visually satisfactory, and the average RANSAC registration error for all of the frames in the map were recorded.

The starting point of the cart was located in the map through manual measures, and the path followed by the cart was marked on the floor to be used as ground truth. The speed of the cart was same as normal walking speed, about $1.5 \mathrm{~m} / \mathrm{s}$. Going faster caused blur in Kinect-captured image, and registration could not be performed. 
Figure 4.7 shows the highlighted ground truth and the path estimated by the system in the ECE Department Office model. A gap can be noticed in the estimated path. The cause of this was incorrect location image retrieval due to either focusing the camera towards a feature-less area, or image blur caused by fast movement speed. Where the path is visible in the figure, 3D registration was being performed. The transformation matrix was estimated between each 3D frame using RANSAC on the feature correspondences, and the re-projection error was recorded.

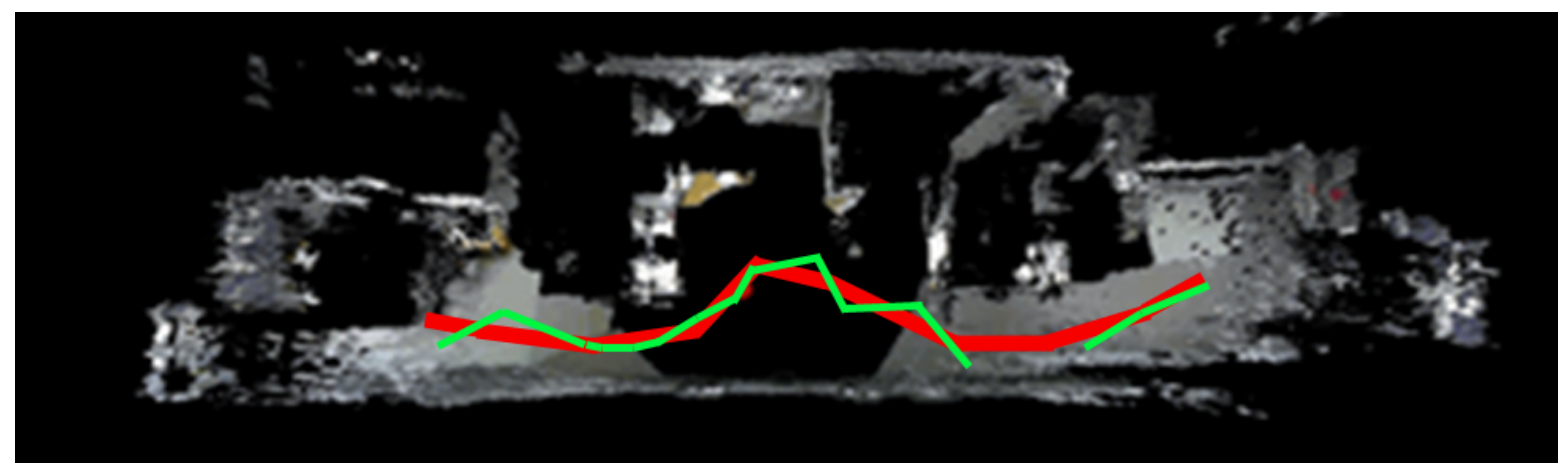

Figure 4.7: The indoor navigation results in the Queen's ECE Dept. office. The red line is ground truth, and the green line highlights the estimated path.

The indoor navigation system is used in two environments:

1. Queen's University ECE Department office;

2. GeoDigital Ottawa Lobby.

Table 4.4 shows the average 3D re-projection error for each of the datasets. The 3D registrations performed during building the map and during navigation followed the same mechanism, so similar errors were observed in both phases. These errors conform with those recorded in the evaluation [17] of the RGBDSLAM system being used with a Kinect. 
Table 4.4: RANSAC Registration Error in Navigation

\begin{tabular}{|c|c|}
\hline Dataset Name & Average Registration Error in RANSAC \\
\hline ECE Dept. Office & $0.173 \mathrm{~m}$ \\
\hline GeoDigital Ottawa Lobby & $0.232 \mathrm{~m}$ \\
\hline
\end{tabular}




\section{Chapter 5}

\section{Conclusions and Future Work}

\subsection{Summary of Conclusions}

A novel image retrieval algorithm, landmark indexing, is introduced in this thesis. This algorithm can be applied on overlapping indoor location images. The overlap is necessary because it provides information on the distinctiveness and repeatability of features upon which the landmark indexing method relies.

Recent publications in the field of location image retrieval are mostly extensions of the bag of visual words method. The main drawback of this methods is identified in this thesis, it creates high dimensional term-frequency histograms for each image in database, each histogram having a dimension of the size of the visual word vocabulary created by extracting features from the training images. Some recent publications use an exhaustive feature set method, which reduces the search space by using features instead of images, but not identifying and removing useless features reduces performance. The proposed landmark indexing algorithm reduces the search space mapping similar features to a particular landmark, and removing the features which are not distinctive or repeatable in the environment. Also, it reduces the high-dimensional 
histogram searching of bag of visual words to a linear search in an array by using a voting scheme on landmarks. The method is tested against the exhaustive feature set method of Liang et al. [39] and bag of visual words [66], on both manually captured and online provided datasets. Experimental results show that the landmark indexing method significantly outperforms the other two methods, and is comparable to and sometimes better in accuracy than the other ones. At runtime, depending on the processor performance, retrieval of an image can be executed in 10-50 ms from a map of over hundreds of frames and more than 40k landmark features.

The proposed image retrieval method is applied in a 3D indoor navigation system, and a series of experiments show that it is able to localize an user, in real-time, with a maximum error of about $200 \mathrm{~mm}$. Given the sensor noise of Microsoft Kinect camera, the error is low. Also, using the Kinect camera, which can provide both 2D and 3D data, the implemented navigation system is one of the cheapest alternatives to a complex system involving multiple cameras and costly laser range finders.

\subsection{Future Work}

There are some improvements that can be performed on the system. The search mechanism being used is multiple randomized $k$-d tree provided in the FLANN library. Although there is an automatic algorithm adjustment method in FLANN, both multiple randomized $k$-d tree and hierarchical $k$-means clustering are not always very reliable. Dynamically determining $k$-value and cluster size may achieve improved search performance. Another improvement is possible by introducing hashing in the landmark indexing, so that the search operation can be performed in $\mathrm{O}(1)$ time.

As discussed in Section 4.4, false positive results show flat feature-to-landmark 
match histograms for training images. Determining the nature of the histogram by fitting appropriate distributions can predict false positives, so post-retrieval refinement can be targeted to those cases rather than all results including true positives.

A limitation of the image retrieval is dynamically changing environment. Although during data acquisition, multiple images are taken of the same location, minor changes may affect features, such as, lighting conditions. In every experiment described in this thesis, query and training images were collected at same time of the day, so lighting conditions are assumed to be unchanged. There are other factors in indoor environments like a door hiding or revealing new areas of the map. The solution may be to implement an update procedure to update the landmark index during runtime.

The scalability of the landmark indexing method can also be verified with a bigger dataset, maybe consisting of more than a thousand or 10 thousand images. It can be speculated that while for other image retrieval methods the feature collection would increase linearly, the proposed landmark index will show a logarithmic style of increase because of internal feature matching. 


\section{Bibliography}

[1] Mirza Tahir Ahmed, Matthew N Dailey, Jose Luis Landabaso, and Nicolas Herrero. Robust key frame extraction for 3D reconstruction from video streams. In In VISAPP International Conference on Computer Vision Theory and Applications, pages 231-236, 2010.

[2] Alexandre Alahi, Raphael Ortiz, and Pierre Vandergheynst. FREAK: Fast retina keypoint. In Computer Vision and Pattern Recognition (CVPR), 2012 IEEE Conference on, pages 510-517, June 2012. doi:10.1109/CVPR.2012.6247715.

[3] M.R. Andersen, T. Jensen, P. Lisouski, A.K. Mortensen, M.K. Hansen, T. Gregersen, and P. Ahrendt. Kinect depth sensor evaluation for computer vision applications. Technical report ECE-TR-6, Department of Engineering, Aarhus University, Denmark, 2012. URL: http://eng.au.dk/fileadmin/DJF/ENG/PDF-filer/Tekniske_rapporter/ Technical_Report_ECE-TR-6-samlet.pdf.

[4] Sunil Arya, David M. Mount, Nathan S. Netanyahu, Ruth Silverman, and Angela Y. Wu. An optimal algorithm for approximate nearest neighbor searching fixed dimensions. Journal of the ACM, 45(6):891-923, November 1998. 
URL: http://doi.acm.org/10.1145/293347.293348, doi:10.1145/293347. 293348.

[5] Herbert Bay, Tinne Tuytelaars, and Luc Gool. SURF: Speeded up robust features. In Ale Leonardis, Horst Bischof, and Axel Pinz, editors, Computer Vision - ECCV 2006, volume 3951 of Lecture Notes in Computer Science, pages 404417. Springer Berlin Heidelberg, 2006. URL: http://dx.doi.org/10.1007/ 11744023_32, doi:10.1007/11744023_32.

[6] Jeffrey S. Beis and David G. Lowe. Shape indexing using approximate nearestneighbour search in high-dimensional spaces. In Computer Vision and Pattern Recognition, 1997. Proceedings., 1997 IEEE Computer Society Conference on, pages 1000-1006, Jun 1997. doi:10.1109/CVPR.1997.609451.

[7] Paul J. Besl and Neil D. McKay. A method for registration of 3-D shapes. IEEE Transactions on Pattern Analysis and Machine Intelligence, 14(2):239-256, Feb 1992. doi:10.1109/34.121791.

[8] Gary Bradski. The OpenCV library. Doctor Dobbs Journal, 25(11):120-126, 2000.

[9] Michael Calonder, Vincent Lepetit, and Pascal Fua. Keypoint signatures for fast learning and recognition. In David Forsyth, Philip Torr, and Andrew Zisserman, editors, Computer Vision - ECCV 2008, volume 5302 of Lecture Notes in Computer Science, pages 58-71. Springer Berlin Heidelberg, 2008. URL: http://dx.doi.org/10.1007/978-3-540-88682-2_6, doi:10. 1007/978-3-540-88682-2_6. 
[10] Michael Calonder, Vincent Lepetit, Christoph Strecha, and Pascal Fua. BRIEF: Binary robust independent elementary features. In Kostas Daniilidis, Petros Maragos, and Nikos Paragios, editors, Computer Vision - ECCV 2010, volume 6314 of Lecture Notes in Computer Science, pages 778-792. Springer Berlin Heidelberg, 2010. URL: http://dx.doi.org/10.1007/978-3-642-15561-1_56, doi:10.1007/978-3-642-15561-1_56.

[11] David M. Chen, Georges Baatz, Kevin Köser, Sam S. Tsai, Ramakrishna Vedantham, Timo Pylvanainen, Kimmo Roimela, Xin Chen, Jeff Bach, Marc Pollefeys, Bernd Girod, and Radek Grzeszczuk. City-scale landmark identification on mobile devices. In Computer Vision and Pattern Recognition (CVPR), 2011 IEEE Conference on, pages 737-744, June 2011. doi:10.1109/CVPR.2011.5995610.

[12] George Chen, John Kua, Stephen Shum, Nikhil Naikal, Matthew Carlberg, and Avideh Zakhor. Indoor localization algorithms for a human-operated backpack system. In 3D Data Processing, Visualization, and Transmission. Citeseer, 2010.

[13] David Crandall, Andrew Owens, Noah Snavely, and Dan Huttenlocher. Discretecontinuous optimization for large-scale structure from motion. In Computer Vision and Pattern Recognition (CVPR), 2011 IEEE Conference on, pages 30013008, June 2011. doi:10.1109/CVPR.2011.5995626.

[14] Navneet Dalal and Bill Triggs. Histograms of oriented gradients for human detection. In Computer Vision and Pattern Recognition, 2005. CVPR 2005. IEEE Computer Society Conference on, volume 1, pages 886-893 vol. 1, June 2005. doi:10.1109/CVPR.2005.177. 
[15] Joost de Weijer and Cordelia Schmid. Coloring local feature extraction. In Ale Leonardis, Horst Bischof, and Axel Pinz, editors, Computer Vision ECCV 2006, volume 3952 of Lecture Notes in Computer Science, pages 334348. Springer Berlin Heidelberg, 2006. URL: http://dx.doi.org/10.1007/ 11744047_26, doi:10.1007/11744047_26.

[16] Hao Du, Peter Henry, Xiaofeng Ren, Marvin Cheng, Dan B. Goldman, Steven M. Seitz, and Dieter Fox. Interactive 3D modeling of indoor environments with a consumer depth camera. In Proceedings of the 13th International Conference on Ubiquitous Computing, UbiComp '11, pages 75-84, New York, NY, USA, 2011. ACM. URL: http://doi.acm.org/10.1145/2030112.2030123, doi:10.1145/ 2030112.2030123.

[17] Felix Endres, Jürgen Hess, Nikolas Engelhard, Jürgen Sturm, Daniel Cremers, and Wolfram Burgard. An evaluation of the RGB-D SLAM system. In Robotics and Automation (ICRA), 2012 IEEE International Conference on, pages 16911696, May 2012. doi:10.1109/ICRA.2012.6225199.

[18] David Filliat. A visual bag of words method for interactive qualitative localization and mapping. In Robotics and Automation, 2007 IEEE International Conference on, pages 3921-3926, April 2007. doi:10.1109/ROBOT.2007.364080.

[19] Jan-Michael Frahm, Pierre Fite-Georgel, David Gallup, Tim Johnson, Rahul Raguram, Changchang Wu, Yi-Hung Jen, Enrique Dunn, Brian Clipp, Svetlana Lazebnik, and Marc Pollefeys. Building rome on a cloudless day. In Kostas Daniilidis, Petros Maragos, and Nikos Paragios, editors, Computer Vision - 
ECCV 2010, volume 6314 of Lecture Notes in Computer Science, pages 368381. Springer Berlin Heidelberg, 2010. URL: http://dx.doi.org/10.1007/ 978-3-642-15561-1_27, doi:10.1007/978-3-642-15561-1_27.

[20] Friedrich Fraundorfer, Henrik Stewénius, and David Nistér. A binning scheme for fast hard drive based image search. In Computer Vision and Pattern Recognition, 2007. CVPR '0\%. IEEE Conference on, pages 1-6, June 2007. doi:10.1109/ CVPR.2007.383174.

[21] Jerome H. Friedman, Jon Louis Bentley, and Raphael Ari Finkel. An algorithm for finding best matches in logarithmic expected time. ACM Transactions on Mathematical Software (TOMS), 3(3):209-226, September 1977. URL: http: //doi.acm.org/10.1145/355744.355745, doi:10.1145/355744.355745.

[22] Keinosuke Fukunaga and Patrenahalli M. Narendra. A branch and bound algorithm for computing k-nearest neighbors. Computers, IEEE Transactions on, C-24(7):750-753, July 1975. doi:10.1109/T-C.1975.224297.

[23] Efstratios Gavves and Cees G.M. Snoek. Landmark image retrieval using visual synonyms. In Proceedings of the International Conference on Multimedia, MM '10, pages 1123-1126, New York, NY, USA, 2010. ACM. URL: http://doi. acm.org/10.1145/1873951.1874166, doi:10.1145/1873951.1874166.

[24] Efstratios Gavves, Cees G.M. Snoek, and Arnold W.M. Smeulders. Visual synonyms for landmark image retrieval. Comput. Vis. Image Underst., 116(2):238249, February 2012. URL: http://dx.doi.org/10.1016/j .cviu.2011.10.004, doi:10.1016/j.cviu.2011.10.004. 
[25] Bernd Girod, Vijay Chandrasekhar, David M. Chen, Ngai-Man Cheung, Radek Grzeszczuk, Yuriy Reznik, Gabriel Takacs, Sam S. Tsai, and Ramakrishna Vedantham. Mobile visual search. Signal Processing Magazine, IEEE, 28(4):6176, July 2011. doi:10.1109/MSP.2011.940881.

[26] Giorgio Grisetti, Slawomir Grzonka, Cyrill Stachniss, Patrick Pfaff, and Wolfram Burgard. Efficient estimation of accurate maximum likelihood maps in 3D. In Intelligent Robots and Systems, 200\%. IROS 200\%. IEEE/RSJ International Conference on, pages 3472-3478, Oct 2007. doi:10.1109/IROS.2007.4399030.

[27] Giorgio Grisetti, Cyrill Stachniss, Slawomir Grzonka, and Wolfram Burgard. A tree parameterization for efficiently computing maximum likelihood maps using gradient descent. In Proceedings of Robotics: Science and Systems (RSS), 2007.

[28] Chris Harris and Mike Stephens. A combined corner and edge detector. In In Proceedings of Fourth Alvey Vision Conference, volume 15, pages 147-151. Manchester, UK, 1988.

[29] Richard Hartley and Andrew Zisserman. Multiple View Geometry in Computer Vision. Cambridge University Press, New York, NY, USA, 2 edition, 2003.

[30] Peter Henry, Michael Krainin, Evan Herbst, Xiaofeng Ren, and Dieter Fox. RGBD mapping: Using kinect-style depth cameras for dense 3D modeling of indoor environments. Int. J. Rob. Res., 31(5):647-663, April 2012. URL: http://dx. doi.org/10.1177/0278364911434148, doi:10.1177/0278364911434148.

[31] Peter Henry, Michael Krainin, Evan Herbst, Xiaofeng Ren, and Dieter Fox. RGB-D mapping: Using kinect-style depth cameras for dense 3D modeling 
of indoor environments. volume 31, pages 647-663, Thousand Oaks, CA, USA, April 2012. Sage Publications, Inc. URL: http://dx.doi.org/10.1177/ 0278364911434148 , doi:10.1177/0278364911434148.

[32] Hervé Jégou, Matthijs Douze, and Cordelia Schmid. Hamming embedding and weak geometric consistency for large scale image search. In Proceedings of the 10th European Conference on Computer Vision: Part I, ECCV '08, pages 304317, Berlin, Heidelberg, 2008. Springer-Verlag. URL: http://dx.doi.org/10. 1007/978-3-540-88682-2_24, doi:10.1007/978-3-540-88682-2_24.

[33] Yan Ke and Rahul Sukthankar. PCA-SIFT: a more distinctive representation for local image descriptors. In Computer Vision and Pattern Recognition, 2004. CVPR 2004. Proceedings of the 2004 IEEE Computer Society Conference on, volume 2, pages II-506-II-513 Vol.2, June 2004. doi:10.1109/CVPR.2004. 1315206.

[34] Jan Knopp, Josef Sivic, and Tomas Pajdla. Avoiding confusing features in place recognition. In Kostas Daniilidis, Petros Maragos, and Nikos Paragios, editors, Computer Vision - ECCV 2010, volume 6311 of Lecture Notes in Computer Science, pages 748-761. Springer Berlin Heidelberg, 2010. URL: http://dx. doi. org/10.1007/978-3-642-15549-9_54, doi:10.1007/978-3-642-15549-9_54.

[35] Kurt Konolige. Sparse sparse bundle adjustment. In Proceedings of the British Machine Vision Conference, pages 102.1-102.11. BMVA Press, 2010. doi:10. 5244/C.24.102. 
[36] Ioannis Kostavelis and Antonios Gasteratos. Learning spatially semantic representations for cognitive robot navigation. Robotics and Autonomous Systems, 61(12):1460-1475, December 2013. URL: http://dx.doi.org/10.1016/ j.robot.2013.07.008, doi:10.1016/j.robot.2013.07.008.

[37] Rainer Kummerle, Giorgio Grisetti, Hauke Strasdat, Kurt Konolige, and Wolfram Burgard. G2o: A general framework for graph optimization. In Robotics and Automation (ICRA), 2011 IEEE International Conference on, pages 3607-3613, May 2011. doi:10.1109/ICRA.2011.5979949.

[38] Stefan Leutenegger, Margarita Chli, and Roland Yves Siegwart. BRISK: Binary robust invariant scalable keypoints. In Computer Vision (ICCV), 2011 IEEE International Conference on, pages 2548-2555, Nov 2011. doi:10.1109/ICCV. 2011.6126542.

[39] Jason Zhi Liang, Nicholas Corso, Eric Turner, and Avideh Zakhor. Reducedcomplexity data acquisition system for image based localization in indoor environments. In International Conference on Indoor Positioning and Indoor Navigation, volume 28, page 31st, 2013.

[40] Tony Lindeberg. Scale-space theory: A basic tool for analyzing structures at different scales. Journal of applied statistics, 21(1-2):225-270, 1994.

[41] Tony Lindeberg. Feature detection with automatic scale selection. International Journal of Computer Vision, 30(2):79-116, 1998. URL: http://dx.doi.org/ 10.1023/A\%3A1008045108935, doi:10.1023/A:1008045108935. 
[42] Timothy Liu, Matthew Carlberg, George Chen, Jacky Chen, John Kua, and Avideh Zakhor. Indoor localization and visualization using a human-operated backpack system. In Indoor Positioning and Indoor Navigation (IPIN), 2010 International Conference on, pages 1-10, Sept 2010. doi:10.1109/IPIN.2010. 5646820.

[43] Manolis I.A. Lourakis and Antonis A. Argyros. SBA: A software package for generic sparse bundle adjustment. ACM Trans. Math. Softw., 36(1):2:1-2:30, March 2009. URL: http://doi.acm.org/10.1145/1486525.1486527, doi:10. $1145 / 1486525.1486527$.

[44] David G. Lowe. Object recognition from local scale-invariant features. In Computer Vision, 1999. The Proceedings of the Seventh IEEE International Conference on, volume 2, pages 1150-1157 vol.2, 1999. doi:10.1109/ICCV.1999. 790410.

[45] David G. Lowe. Distinctive image features from scale-invariant keypoints. Int. J. Comput. Vision, 60(2):91-110, November 2004. URL: http://dx.doi.org/ 10.1023/B:VISI.0000029664.99615.94, doi:10.1023/B:VISI.0000029664. 99615.94.

[46] James MacQueen. Some methods for classification and analysis of multivariate observations. In Proceedings of the fifth Berkeley Symposium on Mathematical Statistics and Probability, volume 1, page 14. California, USA, 1967.

[47] Krystian Mikolajczyk and Cordelia Schmid. Indexing based on scale invariant interest points. In Computer Vision, 2001. ICCV 2001. Proceedings. Eighth 
IEEE International Conference on, volume 1, pages 525-531 vol.1, 2001. doi: 10.1109/ICCV. 2001.937561.

[48] Hans Peter Moravec. Obstacle Avoidance and Navigation in the Real World by a Seeing Robot Rover. PhD thesis, Stanford, CA, USA, 1980. AAI8024717.

[49] Marius Muja and David G. Lowe. Fast approximate nearest neighbors with automatic algorithm configuration. In In VISAPP International Conference on Computer Vision Theory and Applications, pages 331-340, 2009.

[50] Don Murray and James J. Little. Using real-time stereo vision for mobile robot navigation. Autonomous Robots, 8(2):161-171, 2000. URL: http://dx.doi. org/10.1023/A\%3A1008987612352, doi:10.1023/A:1008987612352.

[51] David Nistér and Henrik Stewénius. Scalable recognition with a vocabulary tree. In Computer Vision and Pattern Recognition, 2006 IEEE Computer Society Conference on, volume 2, pages 2161-2168, 2006. doi:10.1109/CVPR.2006.264.

[52] James Philbin, Ondrej Chum, Michael Isard, Josef Sivic, and Andrew Zisserman. Object retrieval with large vocabularies and fast spatial matching. In Computer Vision and Pattern Recognition, 200\%. CVPR '0\%. IEEE Conference on, pages 1-8, June 2007. doi:10.1109/CVPR.2007.383172.

[53] Morgan Quigley, Ken Conley, Brian Gerkey, Josh Faust, Tully Foote, Jeremy Leibs, Rob Wheeler, and Andrew Y Ng. ROS: an open-source robot operating system. In ICRA workshop on open source software, volume 3, 2009. 
[54] Duncan P. Robertson and Roberto Cipolla. An image-based system for urban navigation. In Proceedings of the British Machine Vision Conference, pages 84.1-84.10. BMVA Press, 2004. doi:10.5244/C.18.84.

[55] Edward Rosten and Tom Drummond. Fusing points and lines for high performance tracking. In Computer Vision, 2005. ICCV 2005. Tenth IEEE International Conference on, volume 2, pages 1508-1515 Vol. 2, Oct 2005. doi: 10.1109/ICCV . 2005.104.

[56] Edward Rosten and Tom Drummond. Machine learning for high-speed corner detection. In Ale Leonardis, Horst Bischof, and Axel Pinz, editors, Computer Vision - ECCV 2006, volume 3951 of Lecture Notes in Computer Science, pages 430-443. Springer Berlin Heidelberg, 2006. URL: http://dx. doi .org/10 .1007/ 11744023_34, doi:10.1007/11744023_34.

[57] Ethan Rublee, Vincent Rabaud, Kurt Konolige, and Gary Bradski. ORB: An efficient alternative to SIFT or SURF. In Computer Vision (ICCV), 2011 IEEE International Conference on, pages 2564-2571, Nov 2011. doi:10.1109/ICCV. 2011.6126544.

[58] Yong Rui, Thomas S. Huang, and Shih-Fu Chang. Image retrieval: Current techniques, promising directions, and open issues. Journal of Visual Communication and Image Representation, 10(1):39 - 62, 1999. URL: http: //www.sciencedirect.com/science/article/pii/S1047320399904133, doi: http://dx.doi.org/10.1006/jvci.1999.0413.

[59] Radu Bogdan Rusu, Nico Blodow, and Michael Beetz. Fast point feature histograms (FPFH) for 3D registration. In Robotics and Automation, 2009. 
ICRA '09. IEEE International Conference on, pages 3212-3217, May 2009. doi:10.1109/ROBOT.2009.5152473.

[60] Radu Bogdan Rusu and Steve Cousins. 3D is here: Point cloud library (PCL). In Robotics and Automation (ICRA), 2011 IEEE International Conference on, pages 1-4, May 2011. doi:10.1109/ICRA.2011.5980567.

[61] Torsten Sattler, Tobias Weyand, Bastian Leibe, and Leif Kobbelt. Image retrieval for image-based localization revisited. In Proceedings of the British Machine Vision Conference, pages 1-12. BMVA Press, 2012. doi:http://dx.doi.org/ $10.5244 / C .26 .76$.

[62] Grant Schindler, Matthew Brown, and Richard Szeliski. City-scale location recognition. In Computer Vision and Pattern Recognition, 200\%. CVPR 'O\%. IEEE Conference on, pages 1-7, June 2007. doi:10.1109/CVPR.2007.383150.

[63] Stephen Se, David Lowe, and Jim Little. Vision-based mobile robot localization and mapping using scale-invariant features. In Robotics and Automation, 2001. Proceedings 2001 ICRA. IEEE International Conference on, volume 2, pages 2051-2058 vol.2, 2001. doi:10.1109/ROBOT. 2001.932909.

[64] Stephen Se, David Lowe, and Jim Little. Mobile robot localization and mapping with uncertainty using scale-invariant visual landmarks. The International Journal of Robotics Research, 21(8):735-758, 2002.

[65] Chanop Silpa-Anan and Richard Hartley. Optimised KD-trees for fast image descriptor matching. In Computer Vision and Pattern Recognition, 2008. CVPR 
2008. IEEE Conference on, pages 1-8, June 2008. doi:10.1109/CVPR.2008. 4587638.

[66] Josef Sivic and Andrew Zisserman. Video google: a text retrieval approach to object matching in videos. In Computer Vision, 2003. Proceedings. Ninth IEEE International Conference on, pages 1470-1477 vol.2, Oct 2003. doi:10.1109/ ICCV. 2003.1238663.

[67] Noah Snavely, Steven M. Seitz, and Richard Szeliski. Photo tourism: Exploring photo collections in 3D. ACM Transactions on Graphics (TOG), 25(3):835846, July 2006. URL: http://doi.acm.org/10.1145/1141911.1141964, doi: $10.1145 / 1141911.1141964$.

[68] Natesh Srinivasan. Feature based landmark extraction for real time visual SLAM. In Advances in Recent Technologies in Communication and Computing (ARTCom), 2010 International Conference on, pages 390-394, Oct 2010. doi:10.1109/ARTCom.2010.10.

[69] Christoph Strecha, Timo Pylvanainen, and Pascal Fua. Dynamic and scalable large scale image reconstruction. In Computer Vision and Pattern Recognition (CVPR), 2010 IEEE Conference on, pages 406-413, June 2010. doi:10.1109/ CVPR. 2010.5540184.

[70] Pierre Tirilly, Vincent Claveau, and Patrick Gros. A review of weighting schemes for bag of visual words image retrieval. Rapport de recherche PI 1927, 2009. URL: http://hal.inria.fr/inria-00380706. 
[71] Bill Triggs, Philip F. McLauchlan, Richard I. Hartley, and Andrew W. Fitzgibbon. Bundle adjustment - a modern synthesis. In Bill Triggs, Andrew Zisserman, and Richard Szeliski, editors, Vision Algorithms: Theory and Practice, volume 1883 of Lecture Notes in Computer Science, pages 298-372. Springer Berlin Heidelberg, 2000. URL: http://dx.doi.org/10.1007/3-540-44480-7_21, doi: 10.1007/3-540-44480-7_21.

[72] Panu Turcot and David G. Lowe. Better matching with fewer features: The selection of useful features in large database recognition problems. In Computer Vision Workshops (ICCV Workshops), 2009 IEEE 12th International Conference on, pages 2109-2116, Sept 2009. doi:10.1109/ICCVW.2009.5457541.

[73] Tinne Tuytelaars and Krystian Mikolajczyk. Local invariant feature detectors: A survey. Foundations and Trends® in Computer Graphics and Vision, 3(3):177-280, July 2008. URL: http://dx.doi .org/10.1561/0600000017, doi:10.1561/0600000017.

[74] Koen E.A. Van de Sande, Theo Gevers, and Cees G.M. Snoek. Evaluating color descriptors for object and scene recognition. Pattern Analysis and Machine Intelligence, IEEE Transactions on, 32(9):1582-1596, Sept 2010. doi:10.1109/ TPAMI . 2009.154.

[75] Jianguo Jack Wang, Gibson Hu, Shoudong Huang, and Gamini Dissanayake. 3D landmarks extraction from a range imager data for SLAM. In Australasian Conference on Robotics and Automation (ACRA), 2009. 
[76] Jun Yang, Yu-Gang Jiang, Alexander G. Hauptmann, and Chong-Wah Ngo. Evaluating bag-of-visual-words representations in scene classification. In Proceedings of the International Workshop on Multimedia Information Retrieval, MIR '07, pages 197-206, New York, NY, USA, 2007. ACM. URL: http: //doi.acm.org/10.1145/1290082.1290111, doi:10.1145/1290082.1290111.

[77] AmirRoshan Zamir and Mubarak Shah. Accurate image localization based on google maps street view. In Kostas Daniilidis, Petros Maragos, and Nikos Paragios, editors, Computer Vision - ECCV 2010, volume 6314 of Lecture Notes in Computer Science, pages 255-268. Springer Berlin Heidelberg, 2010. URL: http://dx.doi.org/10.1007/978-3-642-15561-1_19, doi:10. 1007/978-3-642-15561-1_19.

[78] Wei Zhang and Jana Kosecka. Image based localization in urban environments. In $3 D$ Data Processing, Visualization, and Transmission, Third International Symposium on, pages 33-40, June 2006. doi:10.1109/3DPVT.2006.80.

[79] Yu Zhong. Intrinsic shape signatures: A shape descriptor for 3D object recognition. In Computer Vision Workshops (ICCV Workshops), 2009 IEEE 12th International Conference on, pages 689-696, Sept 2009. doi:10.1109/ICCVW. 2009.5457637. 